\title{
EXPLOITING DARK INFORMATION RESOURCES TO CREATE NEW VALUE ADDED SERVICES TO STUDY EARTH SCIENCE PHENOMENA
}

Rahul Ramachandran NASA/MSFC, Manil Maskey UAH, Xiang Li UAH, Kaylin Bugbee UAH

Project Team:

MSFC/UAH: Patrick Gatlin, Amanda Weigel, JJ Miller, Ajinkya Kulkarni

GSFC: Steve Kempler, Suhung Shen, Chung-Lin Shie, Maksym Petrenko

RPI: Peter Fox, Stefan Zednik, Anirudh Prabhu

NAA Invited Talk: Earth Observing Data Science IGARSS July 10-15, 2016 Beijing, China
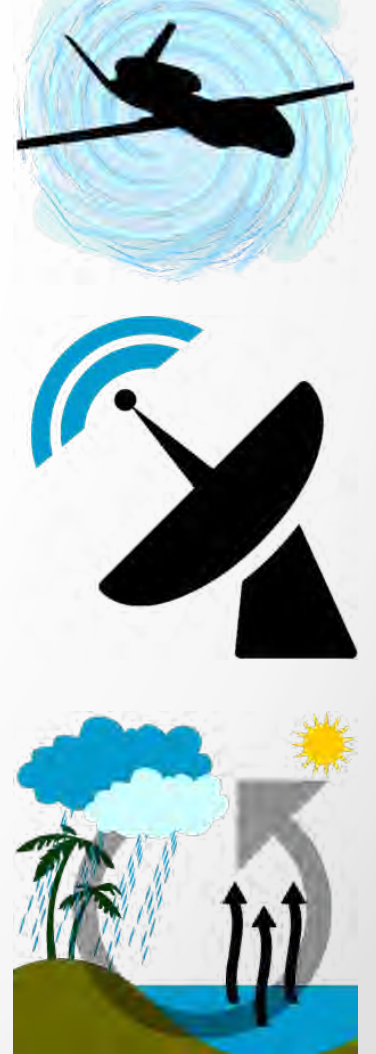


\section{Earth Science Informatics}

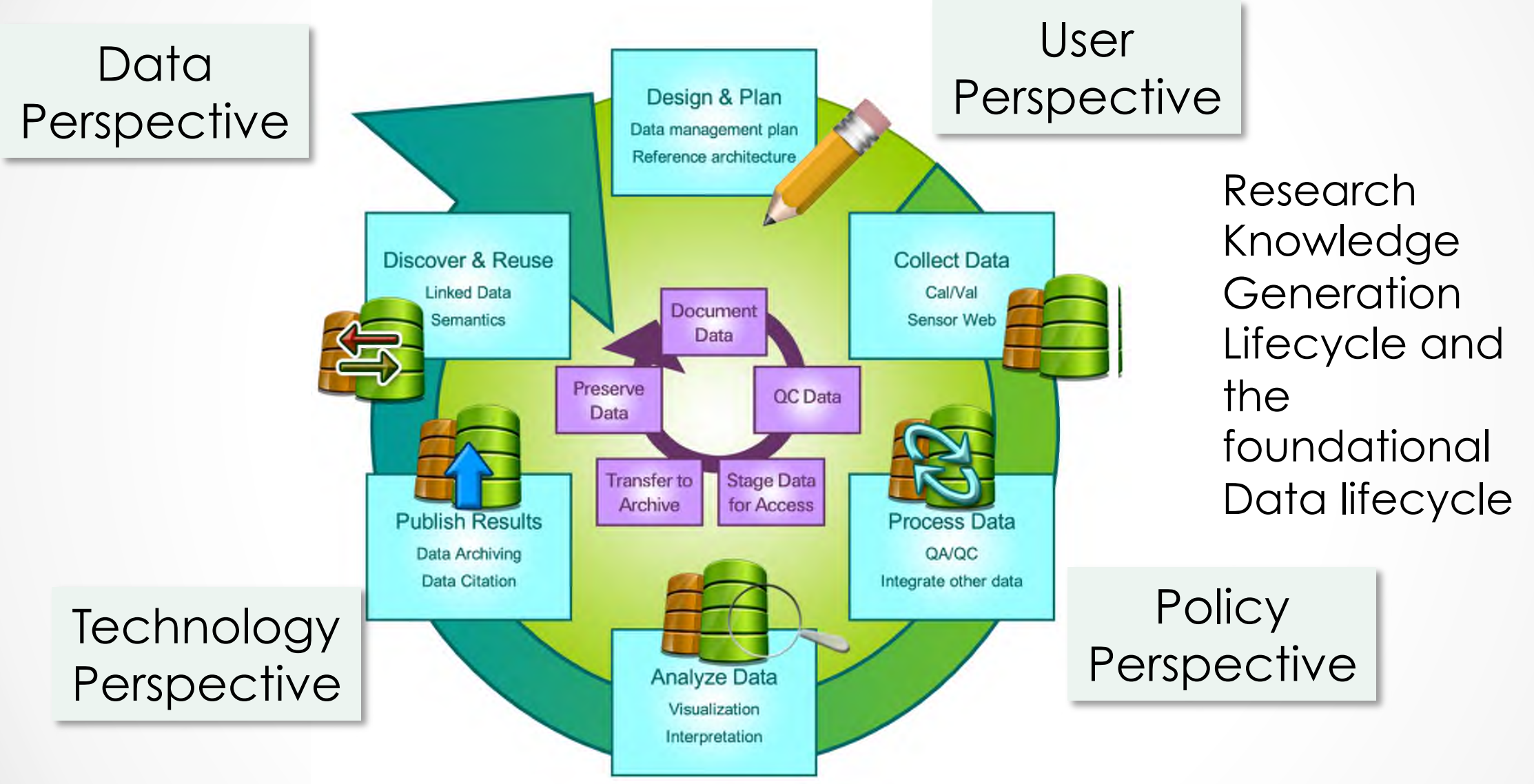

Goals: to make this process efficient, address existing gaps/hurdles, seamlessly integrate new emerging technology, and enable new research capabilities 


\section{Outline}

1. Project Overview

2. Data Curation Service

3. Rules Engine

4. Application (with Demo)

5. Image Retrieval Service

6. Summary 
Part 1: Project Overview 


\section{Motivation}

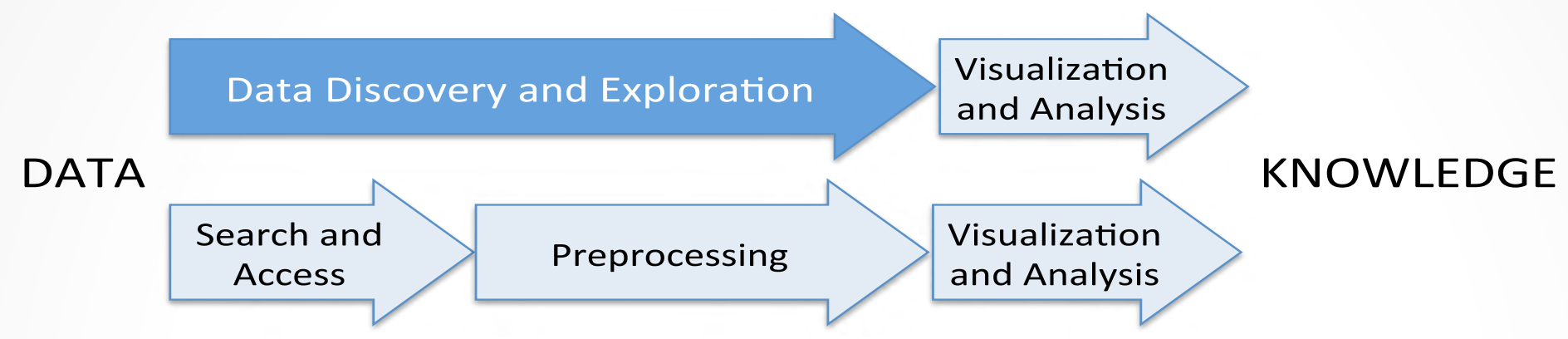

- Data preparation steps are cumbersome and time consuming

- Covers discovery, access and preprocessing

- Limitations of current Data/Information Systems

- Boolean search on data based on instrument or geophysical or other keywords

- Underlying assumption that users have sufficient knowledge of the domain vocabulary

- Lack support for those unfamiliar with the domain vocabulary or the breadth of relevant data available 


\section{Earth Science Metadata: Dark Resources}

- Dark resources - information resources that organizations collect, process, and store for regular business or operational activities but fail to utilize for other purposes

- Challenge is to recognize, identify and effectively utilize these dark data stores

- Metadata catalogs contain dark resources consisting of structured information, free form descriptions of data and browse images.

- NASA's Common Metadata Repository (CMR) holds >6000data collections, 270 million records for individual files and 67 million browse images.

Premise: Metadata catalogs can be utilized beyond their original design intent to provide new data discovery and exploration pathways to support science and education communities. 


\section{Project Goals}

- Design a Semantic Middleware Layer (SML) to exploit these metadata resources

- provide novel data discovery and exploration capabilities that significantly reduce data preparation time.

- utilize a varied set of semantic web, information retrieval and image mining technologies.

- Design SML as a Service Oriented Architecture (SOA) to allow individual components to be used by existing systems

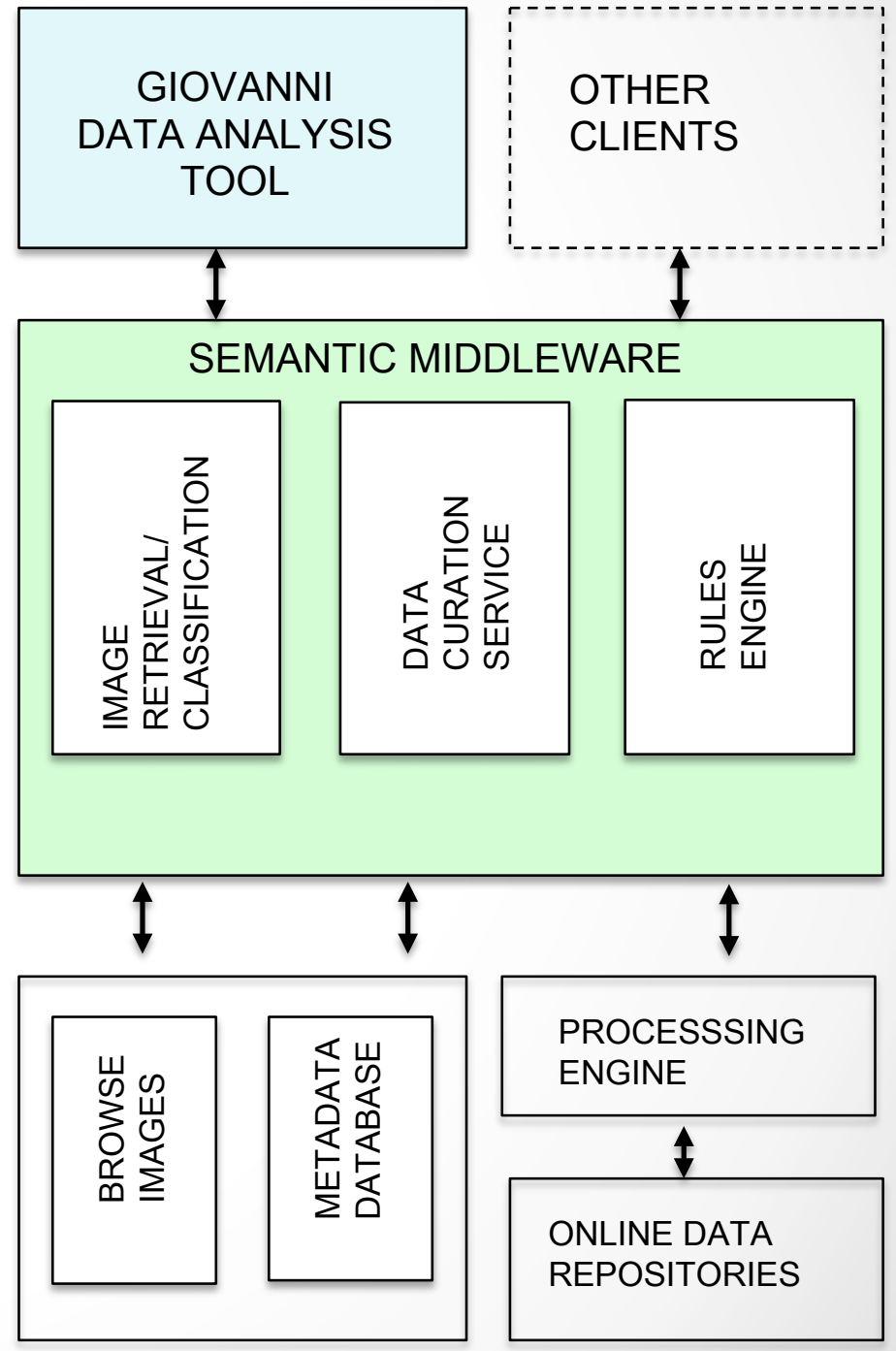




\section{Use Case: \\ Find Interesting Events from Browse Images}

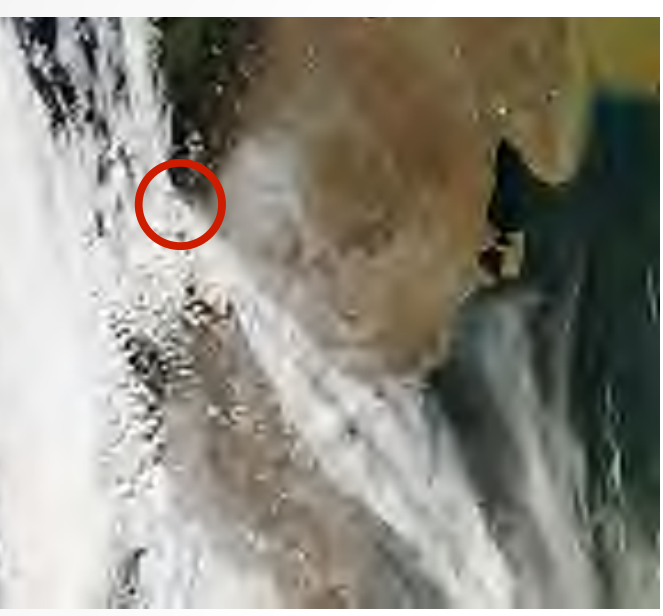

Band 1-4-3 (true color)

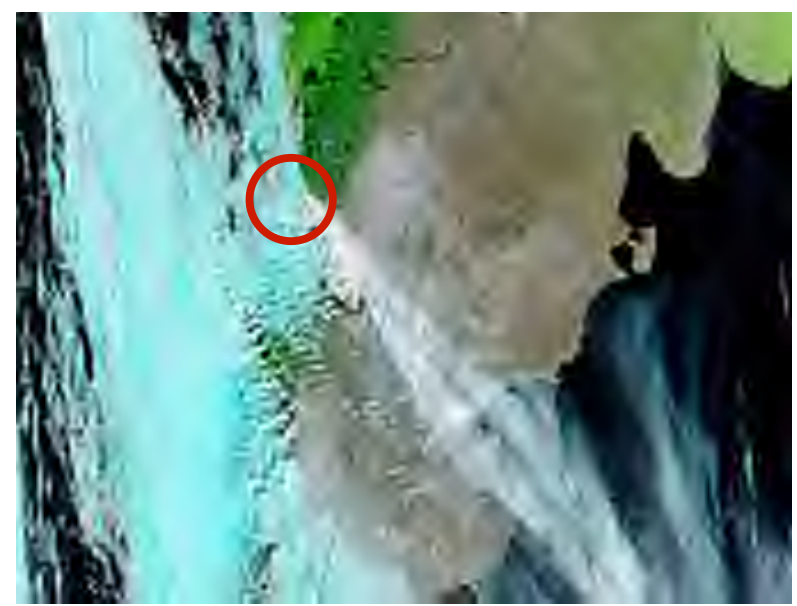

Band 7-2-1

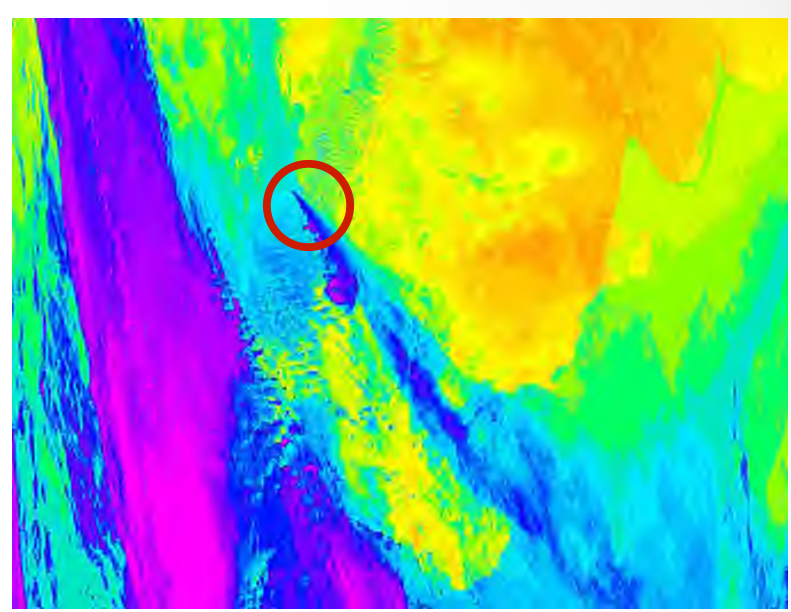

LST

Example: MODIS-Aqua 2008-05-03 18:45 UTC

Chaitén Volcano Eruption

Eruption Time period: May 2 - Nov 2008

Location: Andes region, Chile ( -42.832778 ,

-72.645833)

Image Retrieval Service can be used to find volcanic ash events in browse imagery

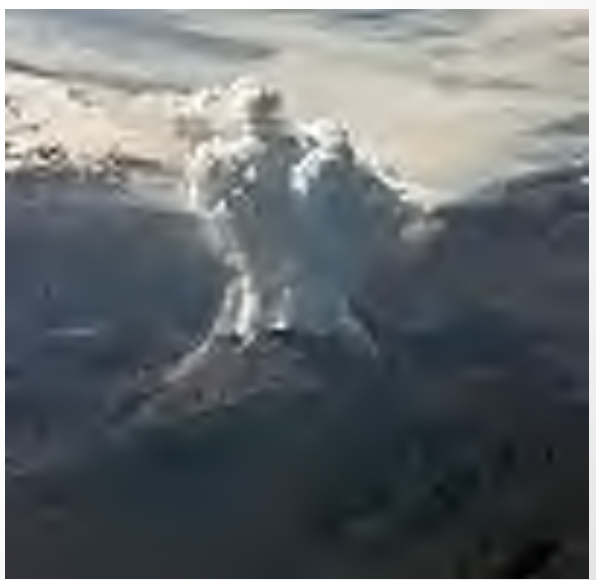




\section{Suggest Relevant Data}

Total $\mathrm{SO}_{2}$ mass:

e.g. Chaitén is $10(\mathrm{kt})=($ kilotons $),(1 \mathrm{kt}=1000$ metric tons $)$

ftp://measures.gsfc.nasa.gov/data/s4pa/SO2/MSVOLSO2L4.1/

MSVOLSO2L4 V01-00-2014m1002.txt

\section{Daily SO2:}

OMI/Aura Sulphur Dioxide (SO2) Total Column Daily L2 Global 0.125 deg http://disc.sci.gsfc.nasa.gov/datacollection/OMSO2G V003.html

\section{Calibrated Radiances:}

MODIS/Aqua Calibrated Radiances 5-Min L1B Swath $1 \mathrm{~km}$

http://dx.doi.org/10.5067/modis/myd021km.006

\section{Aerosol Optical Thickness:}

MODIS/Aqua Aerosol 5-Min L2 Swath 10km

http://modis-atmos.gsfc.nasa.gov/MODC Data Curation Service

SeaWiFS Deep Blue Aerosol Optical Dept recommends relevant

Data $13.5 \mathrm{~km}$

http://disc.gsfc.nasa.gov/datacollection datasets to support event

IR Brightness Temperature: analysis

NCEP/CPC 4-km Global (60 deg N - 60 deg S) Merged IR Brightness

Temperature Dataset 


\section{Generate Giovanni SO2 Plots}

MODIS-Aqua 2008-05-03 18:45 UTC MODIS-Aqua 2008-05-05 18:30 UTC

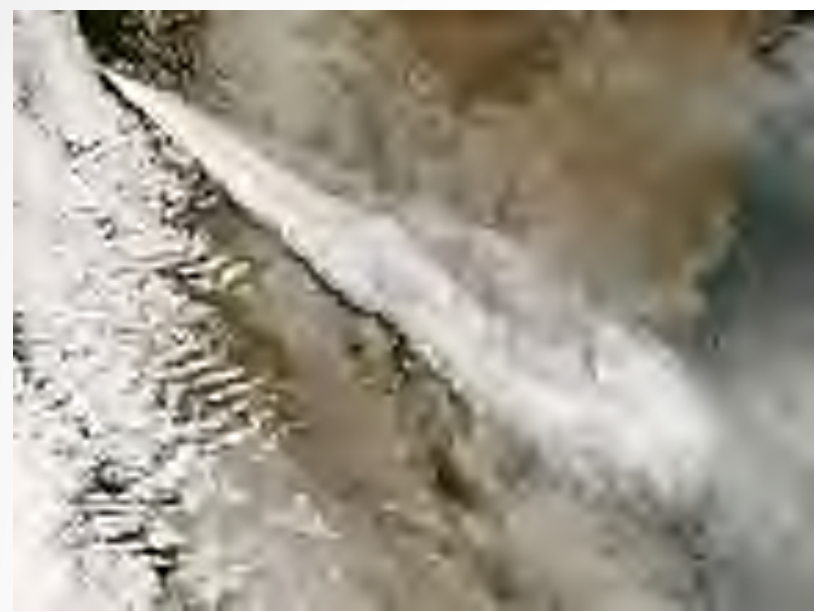

126,003 SO2 Calumn Agrount (Plogetary Boundary La

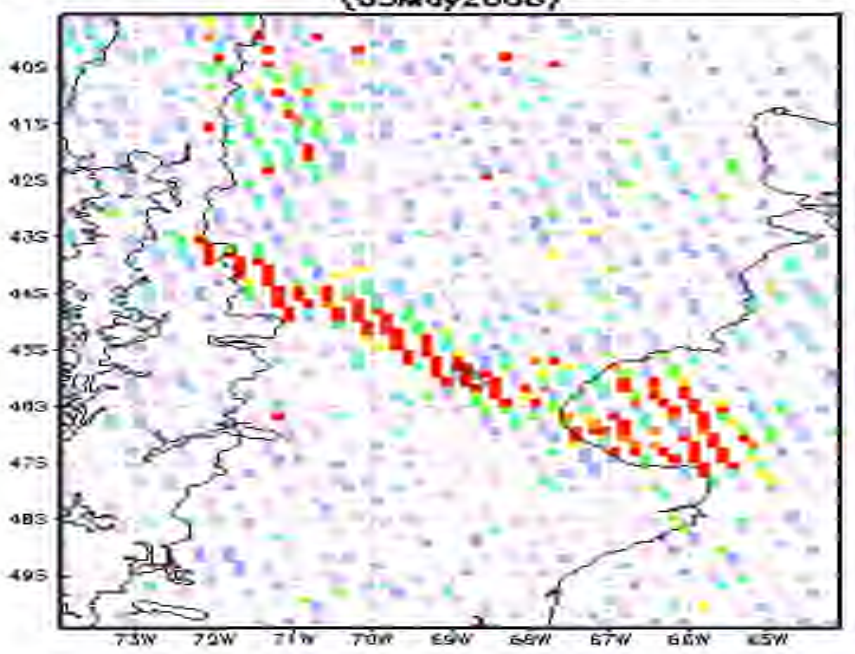

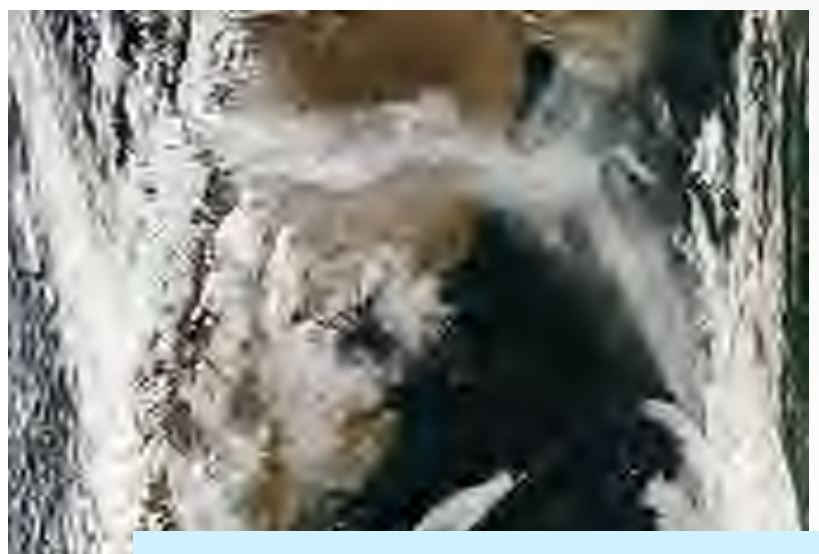

Rules Engine invokes a Giovanni processing workflow to assemble and compare the wind, aerosol and $\mathrm{SO} 2$ data for the event

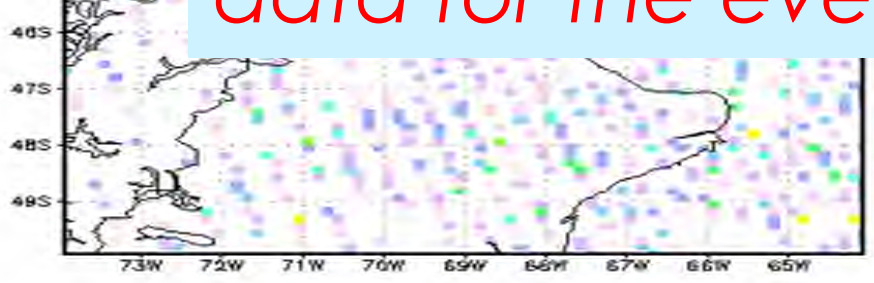

http://gdata2.sci.gsfc.nasa.gov/daac-bin/G3/gui.cgi? instance_id=omil2g 


\section{Conceptual Model}

- Phenomena

- Event type

- Physical Feature

- Manifestation / Driver of phenomena

- Has space/time extent

- Can precede or linger after what is generally thought of as the phenomena event

- Observable Property

- Characteristic/property of physical feature

- Data Variable

- Measurement/estimation of observable feature

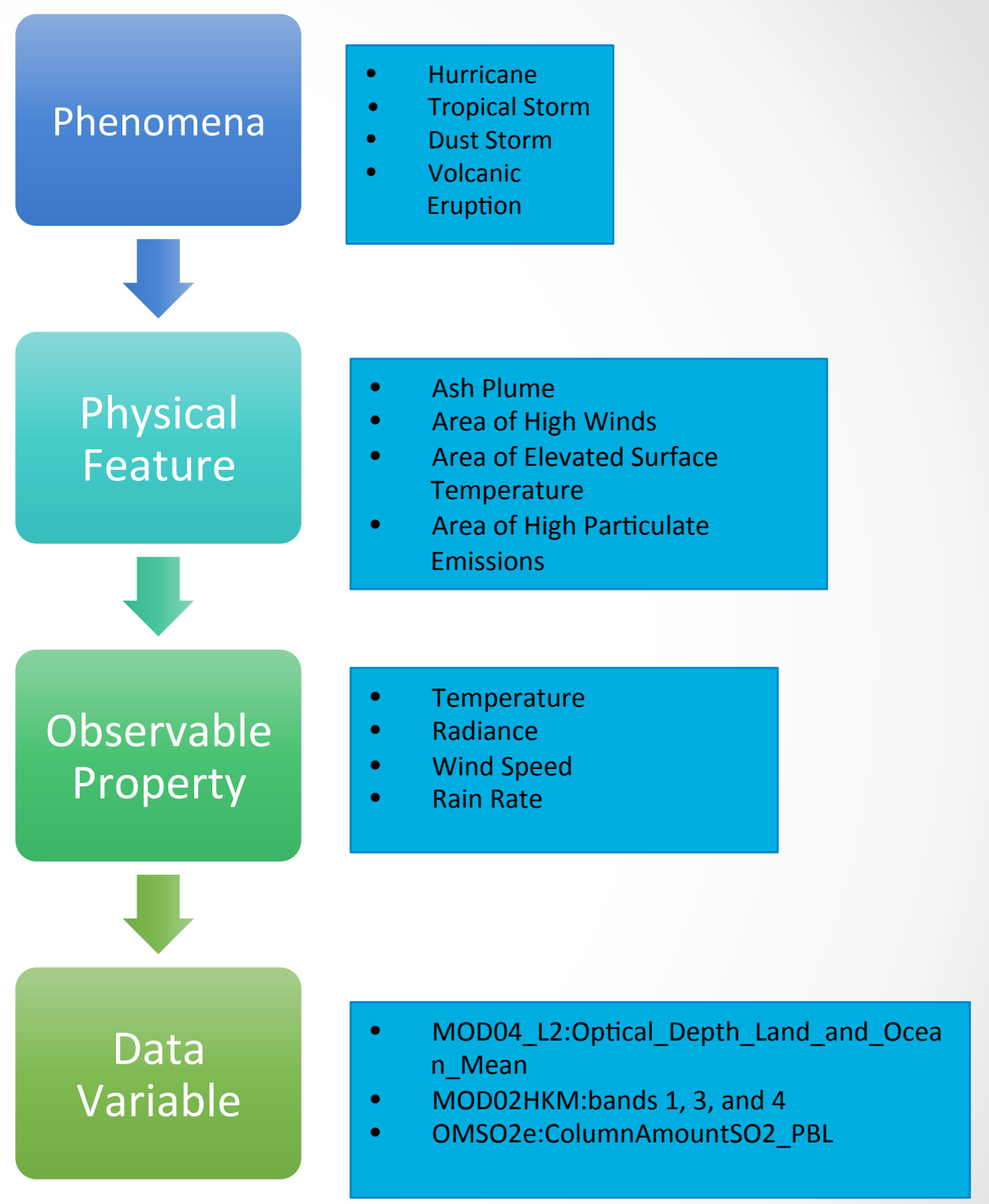


Part 2: Data Curation
Algorithm for Phenomena 


\section{Data Curation}

- Curation is traditionally defined as the process of collecting and organizing information around a common subject matter or a topic of interest and typically occurs in museums, art galleries, and libraries.

- Ramachandran et al. [2015] define geocuration as the act of searching, selecting, and synthesizing Earth science data/metadata and information from across disciplines and repositories into a single, cohesive, and useful collection.

- Manual

- Automated 


\section{Objectives}

- Design a data curation (relevancy ranking) algorithm for a set of phenomena

- Provide the data curation algorithm as a stand alone service

- Envisioned Use:

- Given a phenomenon type (Ex: Hurricane), DCS returns a list of relevant data sets (variables)

- <list of data sets (variables)> = DCS(Phenomenon Type)

- For a specific phenomenon instance (event: Hurricane Katrina), these curated datasets can be filtered based on space/time to get actual granules 


\section{Our Approach}

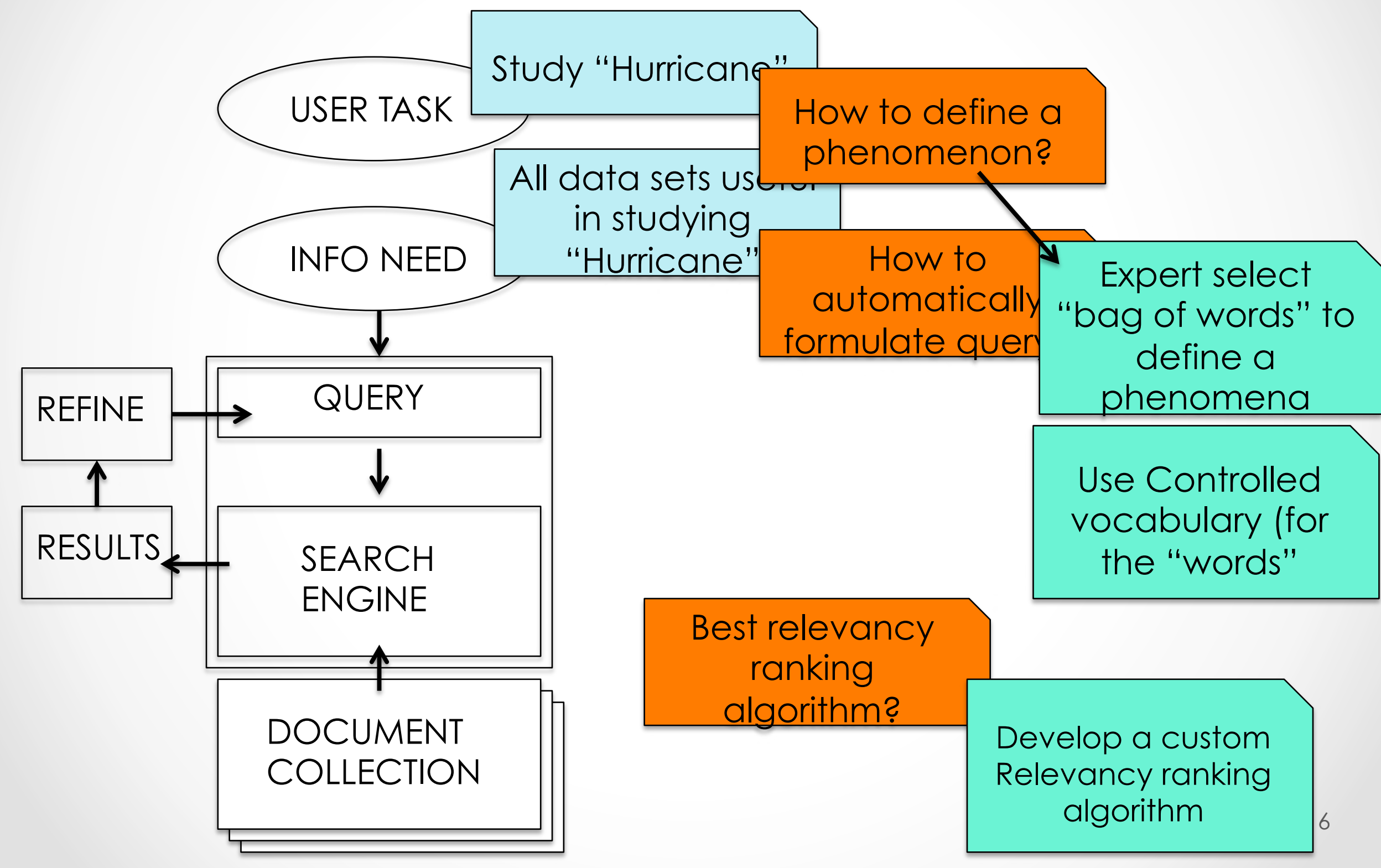




\section{Methodology}

- Selected three metadata fields

- Science Keywords

- Data set long name (title)

- Data set description

- Developed customized vector space model for each field

- Compared different similarity measures

- Cosine vs Jaccard

- Used Weighted Zone Ranking (Ensemble)

o $S_{c}(e)=w_{s} \cdot S_{c}(s)+w_{l} \cdot S_{c}(I)+w_{d} \cdot S_{c}(d)$ 


\section{Experiment Setup}

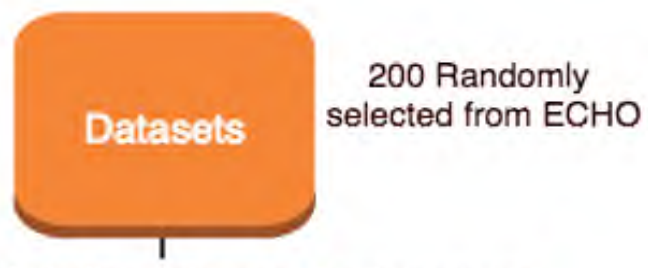

Binary Labeling: relevancy to phenomena
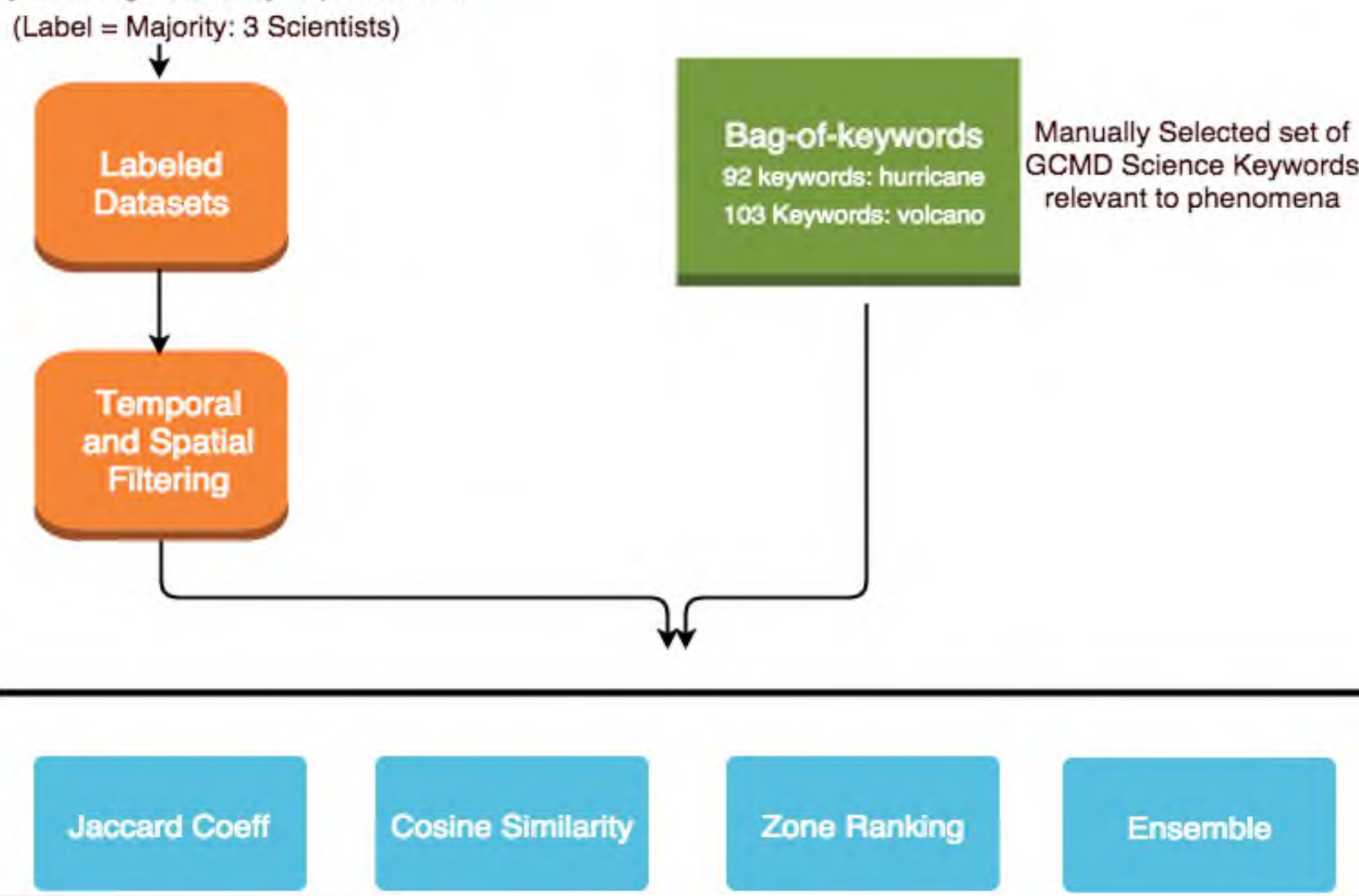

Ensemble 


\section{Comparison of Similarity Measures}

\section{Hurricane}

Jaccard

Coefficient

Top 10

retrieval

Top 20

retrieval

Top 30

retrieval

10

17

23
Volcanic Eruption

Cosine Jaccard Cosine

Similarity Coefficient Similarity

9

6

7

16

15

15

24

22

21

- Both of the measures performed similarly

- Selected Cosine Similarity measure because it is commonly used in space vector model information retrieval 


\section{Ranking Results (Top 20) using Ensemble Method}

\begin{tabular}{|c|c|c|c|c|c|c|}
\hline & \multicolumn{2}{|c|}{ Optimal Weight } & \multicolumn{2}{c|}{ Equal Weight } & \multicolumn{2}{c|}{ Random } \\
\cline { 3 - 7 } & Precision & Recall & Precision & Recall & Precision & Recall \\
\hline Hurricane & $90.0 \%$ & $47.4 \%$ & $85.0 \%$ & $44.7 \%$ & $54.3 \%$ & $28.6 \%$ \\
\hline Volcanic Eruption & $85.0 \%$ & $68.0 \%$ & $80.0 \%$ & $64.0 \%$ & $62.5 \%$ & $50.0 \%$ \\
\hline Fire & $75.0 \%$ & $30.0 \%$ & $75.0 \%$ & $30.0 \%$ & $64.1 \%$ & $25.6 \%$ \\
\hline Flood & $65.0 \%$ & $48.1 \%$ & $55.0 \%$ & $40.7 \%$ & $35.5 \%$ & $26.3 \%$ \\
\hline
\end{tabular}

- Different numbers of "relevant" data sets, collection size (recall) exist within each truth set for each phenomenon

- Better to compare the curation results against the random selection rather than compare the performance against each other

- On average, precision improves about $25 \%$ when using our method and recall improves about 16\% 


\section{Optimal ensemble weights for each phenomenon}

\begin{tabular}{|c|}
\hline Phenomenon \\
\hline Hurricane \\
\hline Volcanic Eruption \\
\hline Fire \\
\hline Flood \\
\hline
\end{tabular}

Optimal Weight Set ( $\left.\mathrm{W}_{\text {sciencekeyword }}, \mathrm{W}_{\text {longname, }}, \mathrm{W}_{\text {description }}\right)$

$(0.6,0.1,0.3)$

$(0.2,0.6,0.2)$

$(0.6,0.2,0.2)$

$(0.5,0.4,0.1)$

- Weight for science keyword is largest while the weight for description is smallest

- Science keywords metadata fields use a controlled vocabulary and should be accurate and consistent

- Description field is free-text and has the most variability in quality 


\section{Methodology Limitations}

- Modeling the search intent is difficult

- one may be interested in only a specific aspect of a phenomenon whereas another user may only be interested in some other characteristic of a phenomenon

- Quality of metadata records is variable

- Key assumptions is that the metadata records stored in the CMR catalog are consistent, correct, and complete

- Launched a project to fix this

- Granularity of the Controlled Vocabulary

- Rich detailed controlled vocabulary provides a better level of annotation granularity to represent different phenomena and help disambiguate data sets

- Truth set labels may be biased

- domain experts on our team have stronger expertise in certain areas such as hurricanes and weaker expertise in others 


\section{Next: Find relevant data fields}

- Need actual data variables

- Example: Giovanni uses these fields for visualization

- What we know

- Data set (Collection) level science keywords (GCMD) - Experts

- Granule data fields and metadata - Auto extract*

- How do we map?

- Start with GCMD to CF Standard name

- Most don't follow CF Standard names 


\section{Approach}

\section{Dataset}

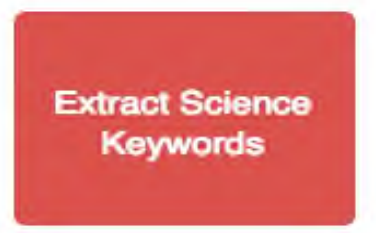

Text processing
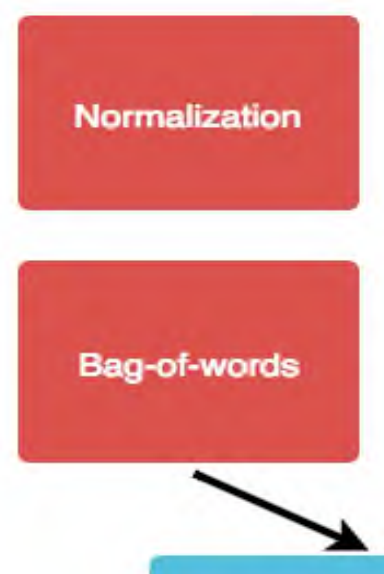

\section{Intersection}

\section{Granules}

Extract Variables and Descriptions

OPeNDAP, netCDF Libs, ...

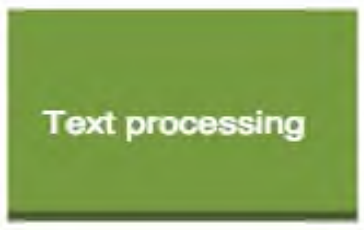

Remove special characters, Tokenize,

$$
\text { ... }
$$

Look up Table

Acronym/Abbreviation expansion, CF

NLP

Learn Patterns

Normalization

Remove stopwords/Stem/Lemmatize

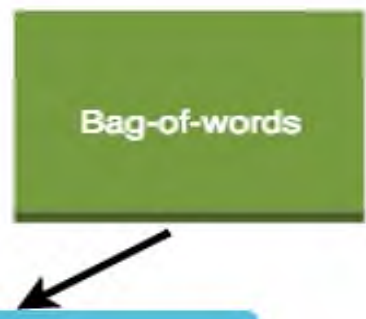

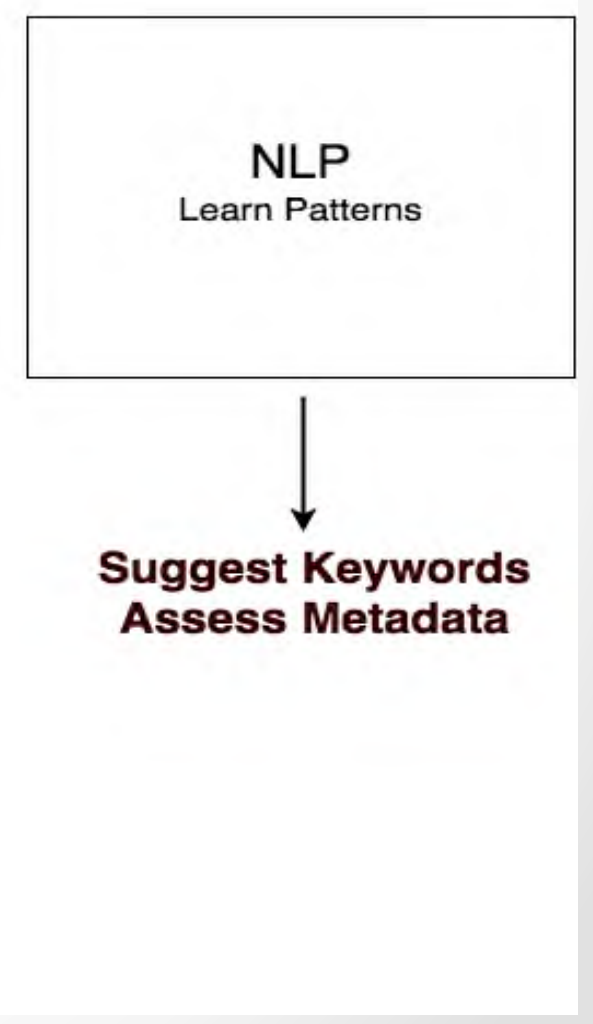




\section{Example: GLAS/ICESat L2 Global Thin Cloud/Aerosol Optical Depths Data (HDF5) V033 - Dataset Metadata}
EARTHDAT $\beta$
GLAS/ICESat L2 Global Thin Cloud/Aer
○ Temporal -
女 Spatial -
A A Feedback

\section{$\Theta$ Back to Granules}

GLAS/ICESat L2 Global Thin Cloud/Aerosol Optical Depths

Data (HDF5) V033 VERSION 33

John.P.Dimarzio.1@nasa.gov

ICESat Science Investigator-led Processing System (I-SIPS)

757-864-1238 (phone)

David.W.Hancock@nasa.gov

NASA DAAC at the National Snow and Ice Data Center 303-492-6199 (phone)

303-492-2468 (fax)

nsidc@nsidc.org

Science Keywords:

Earth Science Atmosphere Clouds

Earth Science Atmosphere Aerosols

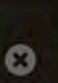

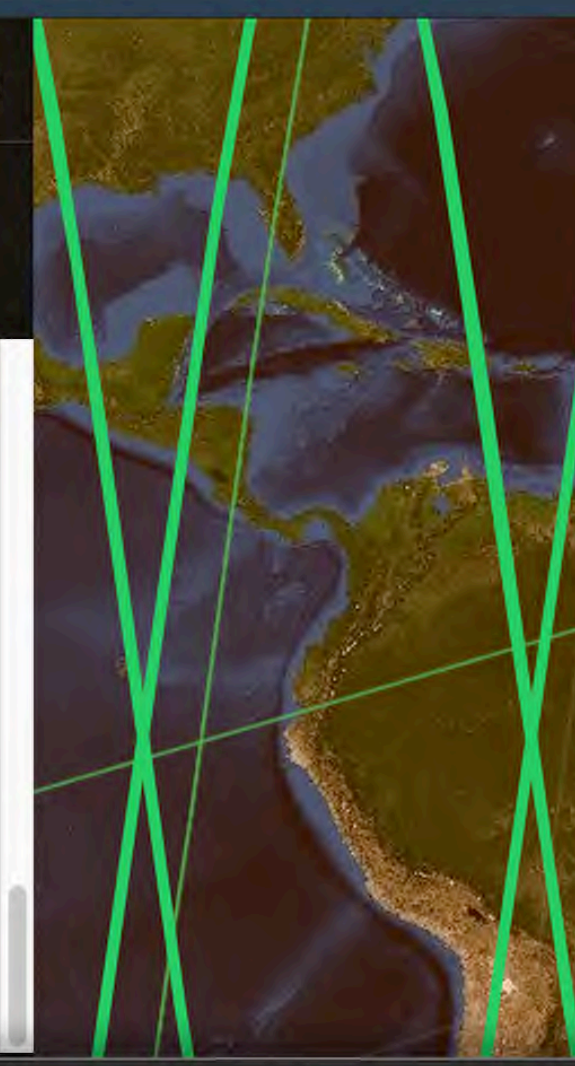

MONTH

$+$

- Aug

GLAS/ICESat L2 Global Thin Cloud/Aerosol Optical Depths Data (HDF5) V033

Sep

Oct

Nov

Dec

Jan

2015
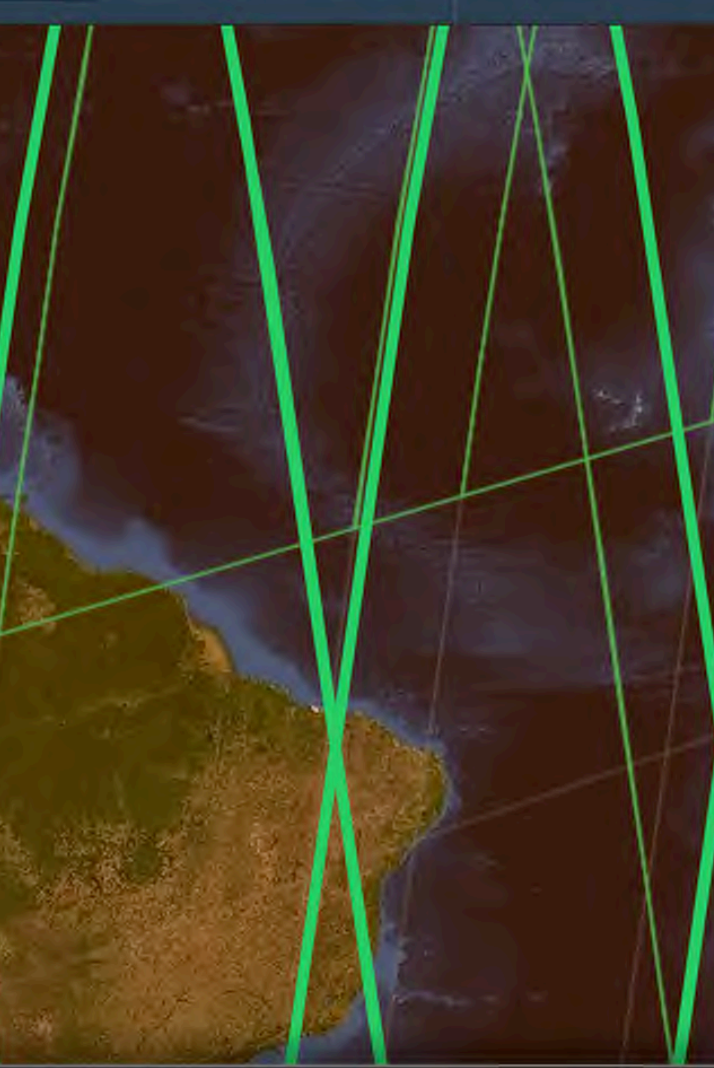


\section{Example: GLAS/ICESat L2 Global Thin Cloud/Aerosol Optical Depths Data (HDF5) V033}

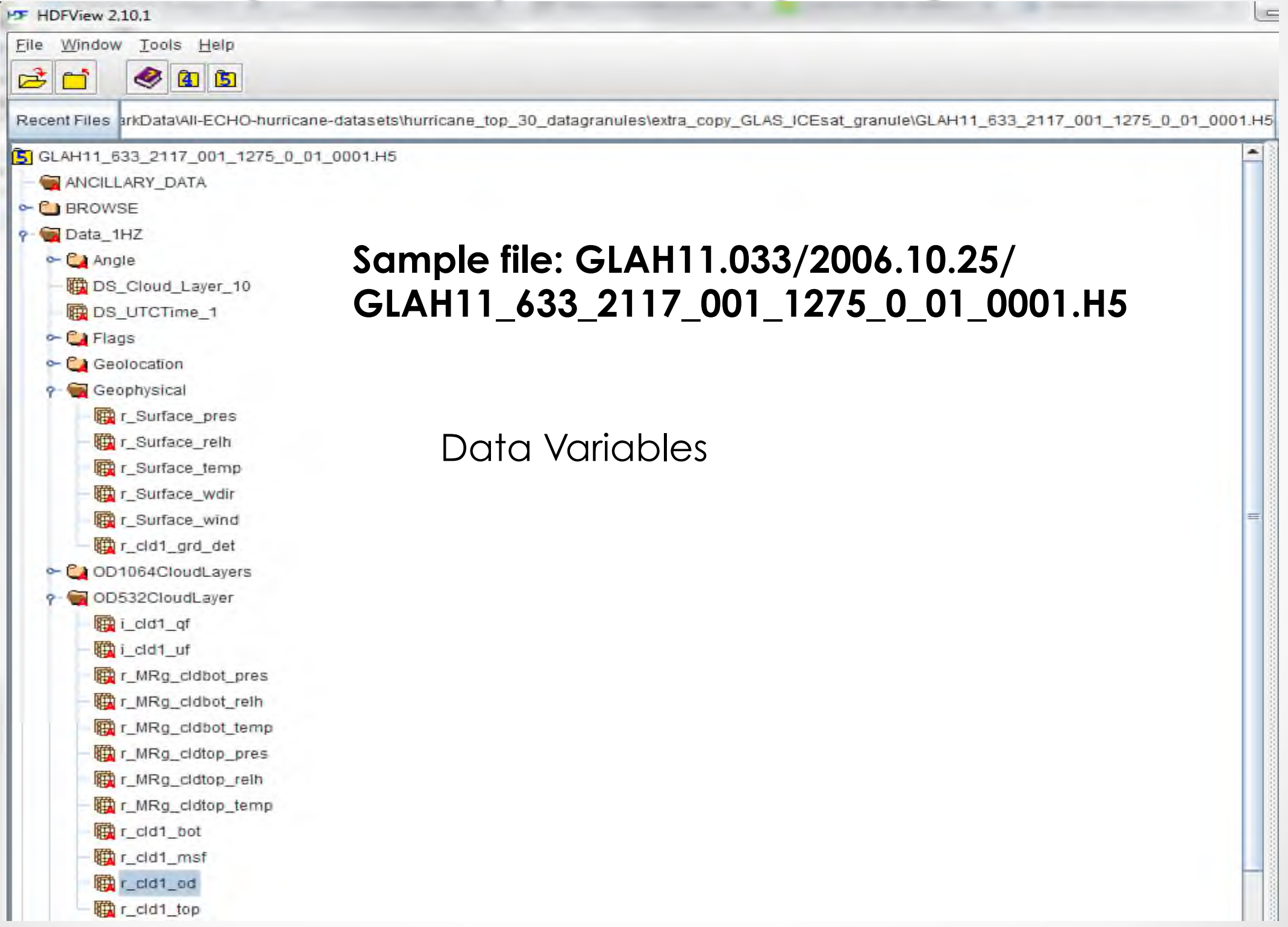

Sample file: GLAH11.033/2006.10.25/ GLAH11_633_2117_001_1275_0_01_0001.H5 


\section{Example: GLASICESat L2 Global Thin Cloud Aerosol Optical Depths Data (HDF5) V033}

\section{Science keyword to variable mapping}

- r_Surface_relh | Surface Relative Humidity

- No match

- r_Surface_temp|Surface Temperature

- No match

- $\quad$ r_Surface_wind|Surface Wind Speed

- No match

- $\quad$ r_cldl_od |Cloud Optical Depth at $532 \mathrm{~nm}$

- Score=3 keyword: ATMOSPHERE->CLOUDS->CLOUD OPTICAL DEPTH/THICKNESS

- Score=2 keyword: ATMOSPHERE->AEROSOLS->AEROSOL OPTICAL DEPTH/THICKNESS

\section{Variable to keyword mapping}

- $\quad$ ATMOSPHERE->CLOUDS->CLOUD OPTICAL DEPTH/THICKNESS

- Score=3 name: r_cld_ir_OD | Cloud Optical Depth at $1064 \mathrm{~nm}$

- score=3 name:i_cld1_af | Cloud optical depth flag for $532 \mathrm{~nm}$

- Score=3 name:i_cld l_uf | Cloud optical depth flag for $532 \mathrm{~nm}$

- Score=3 name:r_cld 1_od | Cloud Optical Depth at $532 \mathrm{~nm}$

- more with low scores

- Serendipitous Discovery - Data Curation Parameter Mapping Algorithm can be used to assess

- Metadata quality for both dataset and granules

- Find incorrect/incomplete keyword annotations

- Automatically suggest science keywords 


\section{Parameter Mapping Tool}

54.172.157.10:5000

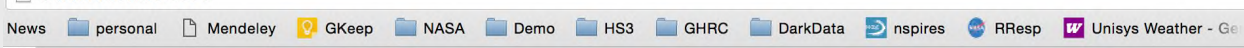

Data Parameter Mapping Tool

\section{Datasets}

AIRS/Aqua Level 2 Support retrieval (AIRS+AMSU) V005

GHRSST Level 2P USA NASA MODIS Aqua SST:1

MODIS/Terra Temperature and Water Vapor Profiles 5-Min L2 Swath $5 \mathrm{~km}$ V005

\section{Datasets}

LIS/OTD 2.5 DEGREE LOW RESOLUTION DIURNAL CLIMATOLOGY (LRDC) V2.3.2013

MODIS/Terra Aerosol 5-Min L2 Swath 10km V005 NRT

MODIS/Terra Aerosol 5-Min L2 Swath 10km V005 NRT

\section{Science Keyword Map}

EDIT

Parameter Map EDIT

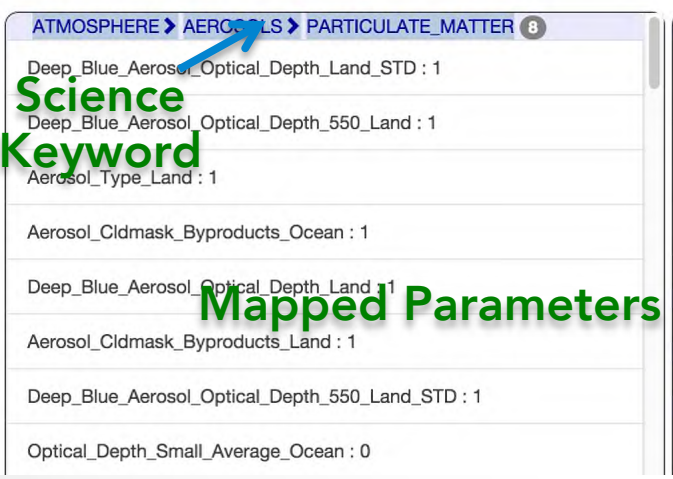

Optical_Depth_Small_Average_Ocean

TMOSPHERE->AEROSOLS-

PAEROSOLS_OPTICAL_DEPTH/THICKNESS : 2 Parameter

ATMOSPHERE->ATMOSPHERIC_RADIATION-

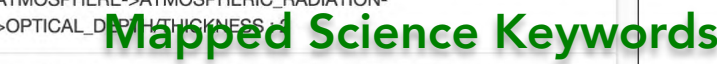

ATMOSPHERE->AEROSOLS->PARTICULATE_MATTER : 0

Asymmetry_Factor_Best_Ocean 0

Deep_Blue_Angstrom_Exponent_Land (0

Cloud_Fraction_Ocean 1

ATMOSPHERE->AEROSOLS->CLOUD_CONDENSATION_NUCLEI : 1
ATMOSPHERE- $\rightarrow$ AEROSOLS- $>$ AEROSOL_PARTICLE_PROPERTIES : 2 ATMOSPHERE- $\rightarrow$ AEROSOLS $\rightarrow$ CLOUD CONDENSATION_NUCLEI :2 ATMOSPHERE- $\rightarrow$ AEROSOLS $\rightarrow$ AEROSOL_EXTINCTION : 2

1. ATMOSPHERE $\rightarrow$ AEROSOLS $\rightarrow$ AEROSOLS_OPTICAL_DEPTHITHICKNESS :2 ATMOSPHERE->AEROSOLS->AEROSOL_RADIANCE: 2

ATMOSPHERE- $>$ AEROSOLS $\rightarrow$ CARBONACEOUS_AEROSOLS : 2

ATMOSPHERE- $\rightarrow$ AEROSOLS- $\rightarrow$ DUST/ASHISMOKE: 2

ATMOSPHERE- $\rightarrow$ AEROSOLS $\rightarrow$ NITRATE_PARTICLES $: 2$ ATMOSPHERE- $>$ AEROSOLS $>$ ORGANIC_PARTICLES : 2

ATMOSPHERE $\rightarrow$ AEROSOLS $\rightarrow$ PARTICULATE_MATTER : 2

ATMOSPHERE- $\rightarrow$ AEROSOLS->SULFATE PARTICLES : 2

ATMOSPHERE- $\rightarrow$ ATMOSPHERIC_RADIATION- $\rightarrow$ RADIATIVE_FLUXX:2

ATMOSPHERE- $\rightarrow$ ATMOSPHERIC_RADIATION $\rightarrow$ REFLECTANCE : 2

$\checkmark$ ATMOSPHERE->ATMOSPHERIC_RADIATION->OPTICAL_DEPTH/THICKNESS : 2

ATMOSPHERE->AEROSOLS-PPARTICULATE_MATTER:0

\section{Mapping Scores Generated by Algorithm}


Part 3: Rules Engine 


\section{What settings should I use to visualize this event?}

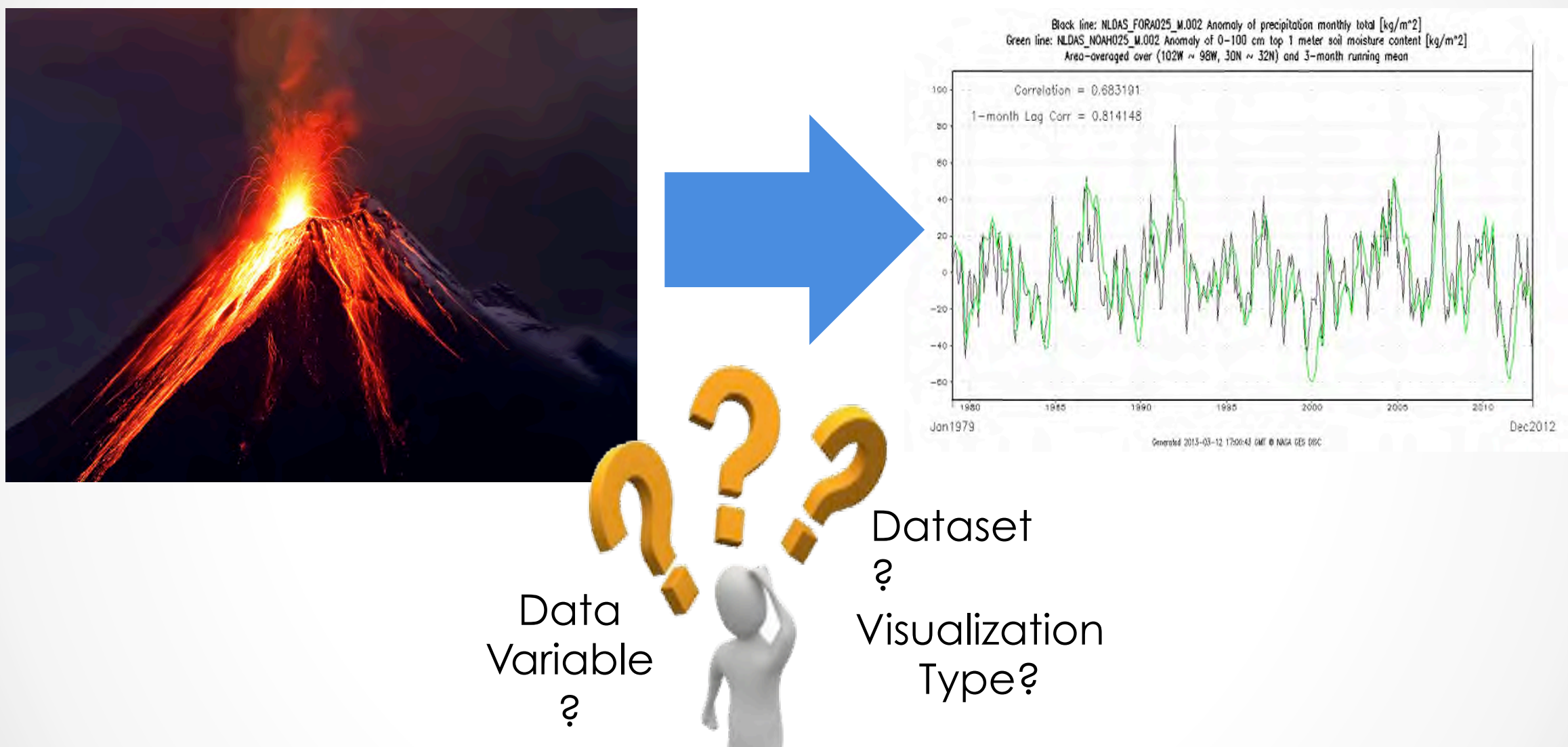

Goal: Automate data preprocessing and exploratory analysis and visualization tasks 


\section{Strategy}

- Service to generate and rank candidate workflow configurations

- Use rules to make assertions about compatibility based on multiple factors

o does this data variable make sense for this feature?

o does this visualization type make sense for this feature?

o does the temporal / spatial resolution of this dataset make sense for this feature?

- Each compatibility assertion type is assigned weights.

$\circ$ ex: Strong $=5$, Some $=3$, Slight $=1$, Indifferent $=0$, Negative $=-1$.

- Based on the aggregated compatibility assertions, we calculate the score for each visualization candidate. 


\section{Ruleset Development}

Survey asked users to rate characteristics of phenomena features

\section{Feature characteristics for analysis *}

What characteristics are of interest when analyzing the feature?

$$
\begin{gathered}
\text { negative indifferent } \begin{array}{l}
\text { slight some strong } \\
\text { value }
\end{array} \text { value value } \\
\text { value }
\end{gathered}
$$

east-west movement

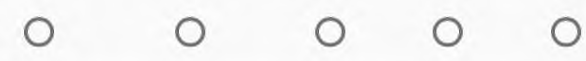

north-south movement

temporal evolution

spatial extent of event

year-to-year variability

may impact seasonal variation

variation with atmospheric height

$\mathrm{O}$

O

0

O

O

height

global phenomena

detection of events
Survey results used to formulate rules

\section{[rule1:}

(?feature rdf:type dd:AshPlume)

$->$

(?feature dd:strongCompatibilityFor dd:temporal_evolution),

(?feature dd: indifferentCompatibilityFor dd:east-west-movement),

] 


\section{Phenomena Feature Characteristic Mappings}

\begin{tabular}{|c|c|c|c|c|c|c|c|c|c|}
\hline Phenomena & $\begin{array}{l}\text { East- } \\
\text { West } \\
\text { Movem } \\
\text { ent }\end{array}$ & $\begin{array}{l}\text { North- } \\
\text { South } \\
\text { Movement }\end{array}$ & $\begin{array}{l}\text { Temporal } \\
\text { Evolution }\end{array}$ & $\begin{array}{l}\text { Spatial } \\
\text { Extent of } \\
\text { Event }\end{array}$ & $\begin{array}{l}\text { Year-to- } \\
\text { Year } \\
\text { Variability }\end{array}$ & $\begin{array}{l}\text { May } \\
\text { Impact } \\
\text { Seasonal } \\
\text { Variation }\end{array}$ & $\begin{array}{l}\text { Variation } \\
\text { with } \\
\text { Atmospher } \\
\text { ic Height }\end{array}$ & $\begin{array}{l}\text { Global } \\
\text { Phenomen } \\
\text { a }\end{array}$ & $\begin{array}{l}\text { Detection } \\
\text { of Events }\end{array}$ \\
\hline $\begin{array}{l}\text { Volcano - } \\
\text { Ash Plume }\end{array}$ & $\begin{array}{l}\text { Indiffere } \\
\text { nt }\end{array}$ & Indifferent & Strong & Slight & Strong & Strong & Strong & Strong & Strong \\
\hline Flood & Some & Some & Strong & Some & Some & Strong & Some & Slight & Some \\
\hline Dust Storm & Strong & Strong & Strong & Strong & Indifferent & Indifferent & Strong & Indifferent & Some \\
\hline
\end{tabular}




\section{Service to Characteristic Mappings}

\begin{tabular}{|c|c|c|c|c|c|c|c|c|c|c|}
\hline Service & $\begin{array}{l}\text { Visualizatio } \\
\text { n }\end{array}$ & $\begin{array}{l}\text { East-West } \\
\text { Movement }\end{array}$ & $\begin{array}{l}\text { North-South } \\
\text { Movement }\end{array}$ & $\begin{array}{l}\text { Temporal } \\
\text { Evolution }\end{array}$ & $\begin{array}{l}\text { Spatial } \\
\text { Extent of } \\
\text { Event }\end{array}$ & $\begin{array}{l}\text { Year-to- } \\
\text { Year } \\
\text { Variability }\end{array}$ & $\begin{array}{l}\text { Seasonal } \\
\text { Variation }\end{array}$ & $\begin{array}{l}\text { Variation } \\
\text { with } \\
\text { Atmospheri } \\
\text { c Height }\end{array}$ & $\begin{array}{l}\text { Global } \\
\text { Phenomena }\end{array}$ & $\begin{array}{l}\text { Detection of } \\
\text { Events }\end{array}$ \\
\hline $\begin{array}{l}\text { Time- } \\
\text { averaged } \\
\text { Map }\end{array}$ & $\begin{array}{c}\text { Color-Slice } \\
\text { Map }\end{array}$ & & & & & & & & & \\
\hline $\begin{array}{c}\text { Area- } \\
\text { averaged } \\
\text { Time Series }\end{array}$ & Time Series & & & & & & & & & \\
\hline $\begin{array}{c}\text { User- } \\
\text { defined } \\
\text { Climatology }\end{array}$ & $\begin{array}{c}\text { Color-Slice } \\
\text { Map }\end{array}$ & & & & & & & & & \\
\hline $\begin{array}{l}\text { Vertical } \\
\text { Profile }\end{array}$ & Line Plot & & & & & & & & & \\
\hline $\begin{array}{l}\text { Seasonal } \\
\text { Time Series }\end{array}$ & Time Series & & & & & & & & & \\
\hline $\begin{array}{l}\text { Zonal } \\
\text { Means }\end{array}$ & Line Plot & & & & & & & & & \\
\hline $\begin{array}{l}\text { Hovmoller } \\
\text { (Longitude) }\end{array}$ & $\begin{array}{c}\text { Color-Slice } \\
\text { Grid }\end{array}$ & & & & & & & & & \\
\hline $\begin{array}{l}\text { Hovmoller } \\
\text { (Latitude) }\end{array}$ & $\begin{array}{c}\text { Color-Slice } \\
\text { Grid }\end{array}$ & & & & & & & & & \\
\hline
\end{tabular}




\section{Compute Compatibility}
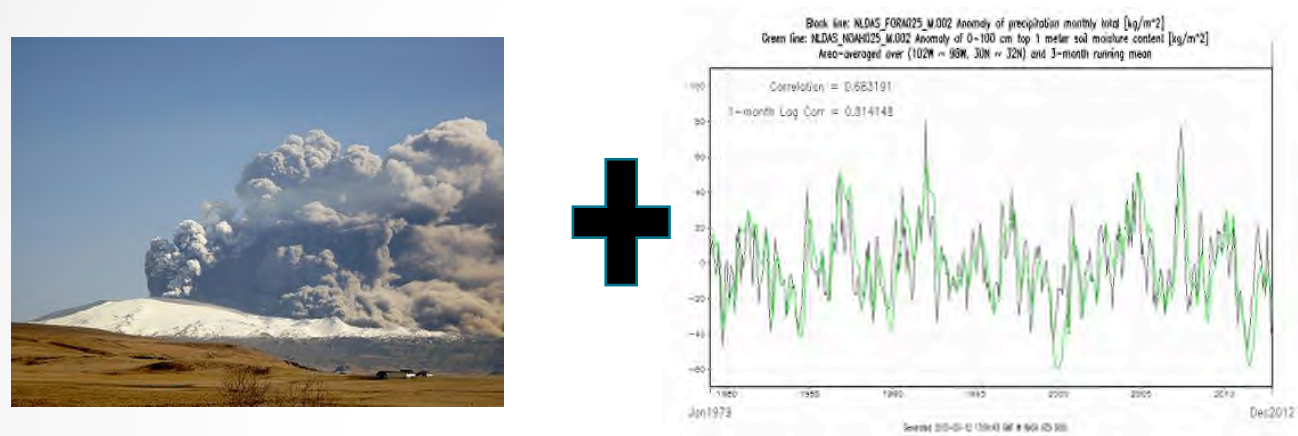

Phenomena:

Volcano - Ash

Plume

\begin{tabular}{|l|l|}
\hline $\begin{array}{l}\text { Temporal } \\
\text { Evolution }\end{array}$ & $\begin{array}{l}\text { Detectio } \\
\mathrm{n} \text { of } \\
\text { Events }\end{array}$ \\
\hline Strong & Strong \\
\hline
\end{tabular}

Service - Area

Averaged Time

Series

\begin{tabular}{|l|l|}
\hline Area & Temporal \\
Averaged & evolution; \\
Time Series : & Detection \\
bestFor $\rightarrow$ & of events \\
\hline
\end{tabular}

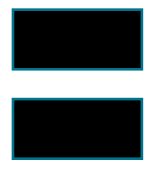

STRONG

COMPATIBILITY

$\times 2$

Images from , htto://disc.sci.asfc.nasa.aov/datareleases/images/nldas monthlv climatoloav fiaure 9.aif, http://www.clipartbest.com/cliparts/biy/bAX/biybAXGil.png 
Part 4: Application (Demo) 


\section{Integrating Services in}

\section{Giovanni}

- Tool: Giovanni is a popular on-line environment that lets users discover, plot, and download a number of geophysical parameters (data variables)

- Goal: Leverage Dark Data services and technologies to assist Giovanni users in discovering and exploring data

'Success will be realized when Giovanni requests can be automatically invoked with the appropriate spatial and temporal extents, variables and workflow / visualization type for a particular event'

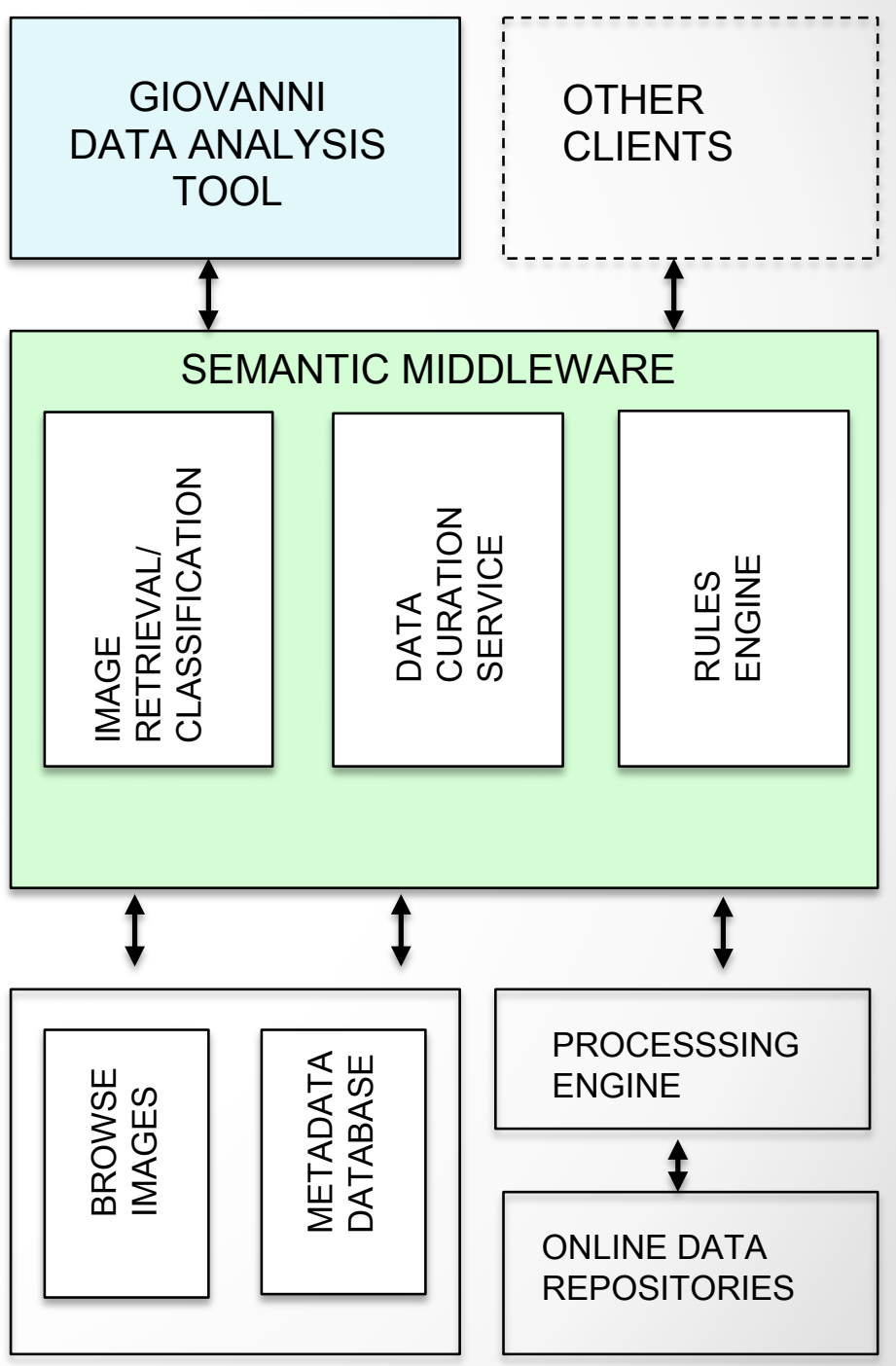




\section{Giovanni - Standard Edition}

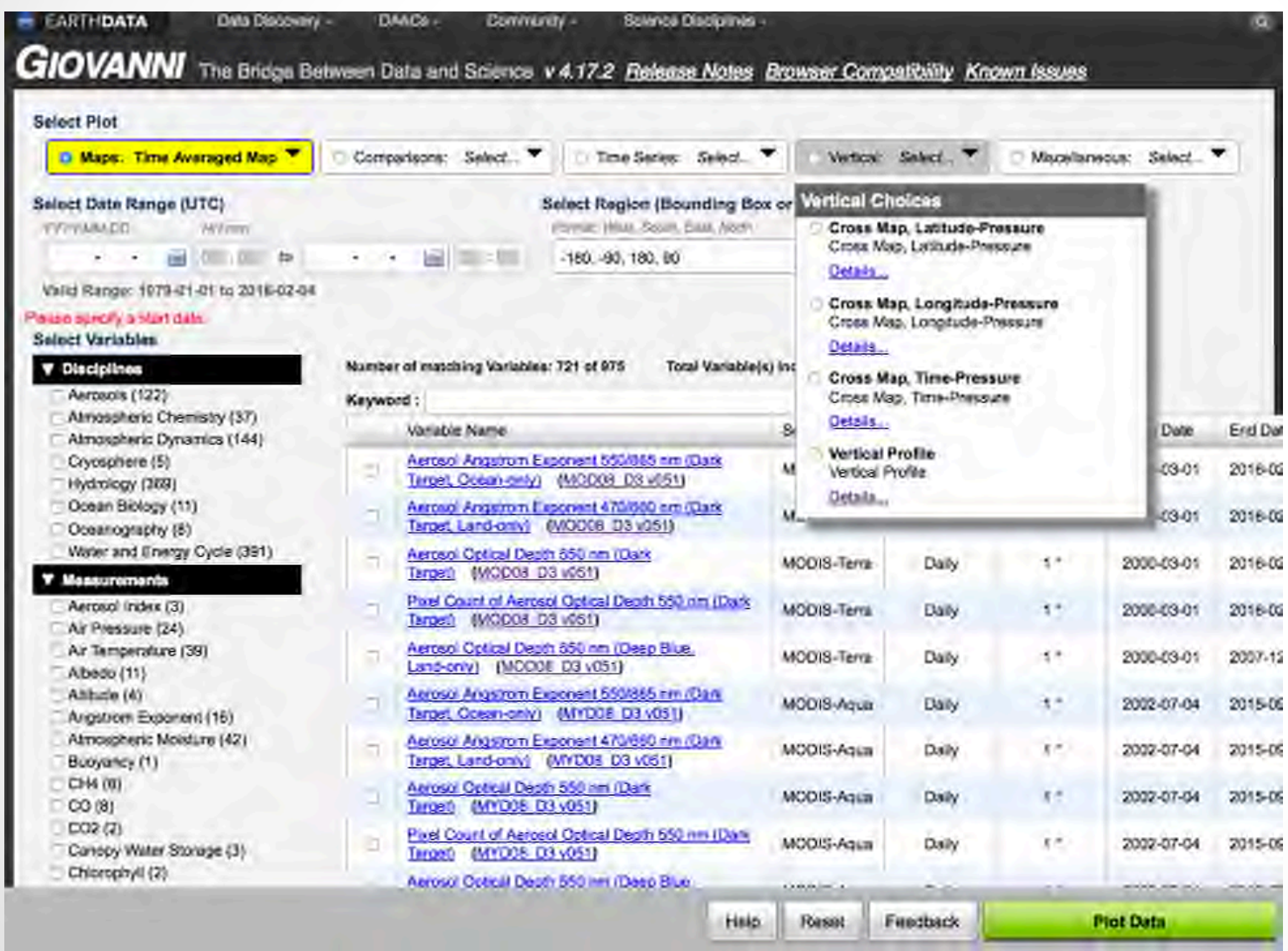

User needs to decide:

- Variable(s)

- Time

- Space

- Plot type 


\section{Giovanni - Dark Data Edition}

Rules Service:

highlights

suitable plots

based on

selected event

\& variables

Curation

Service: event

type filters

relevant

variables
Selected event \& its time Event Client

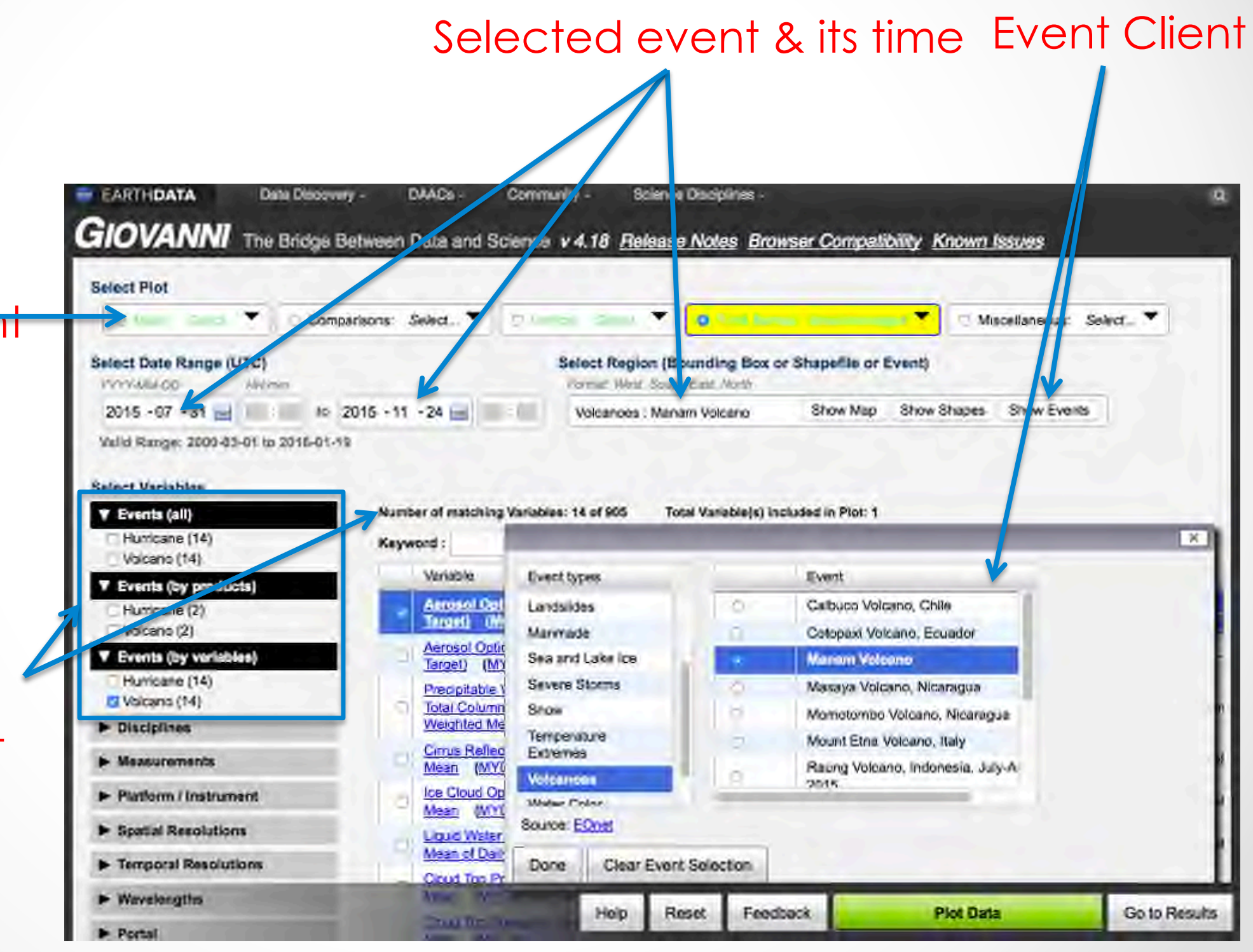




\section{Giovanni - Dark Data Edition}

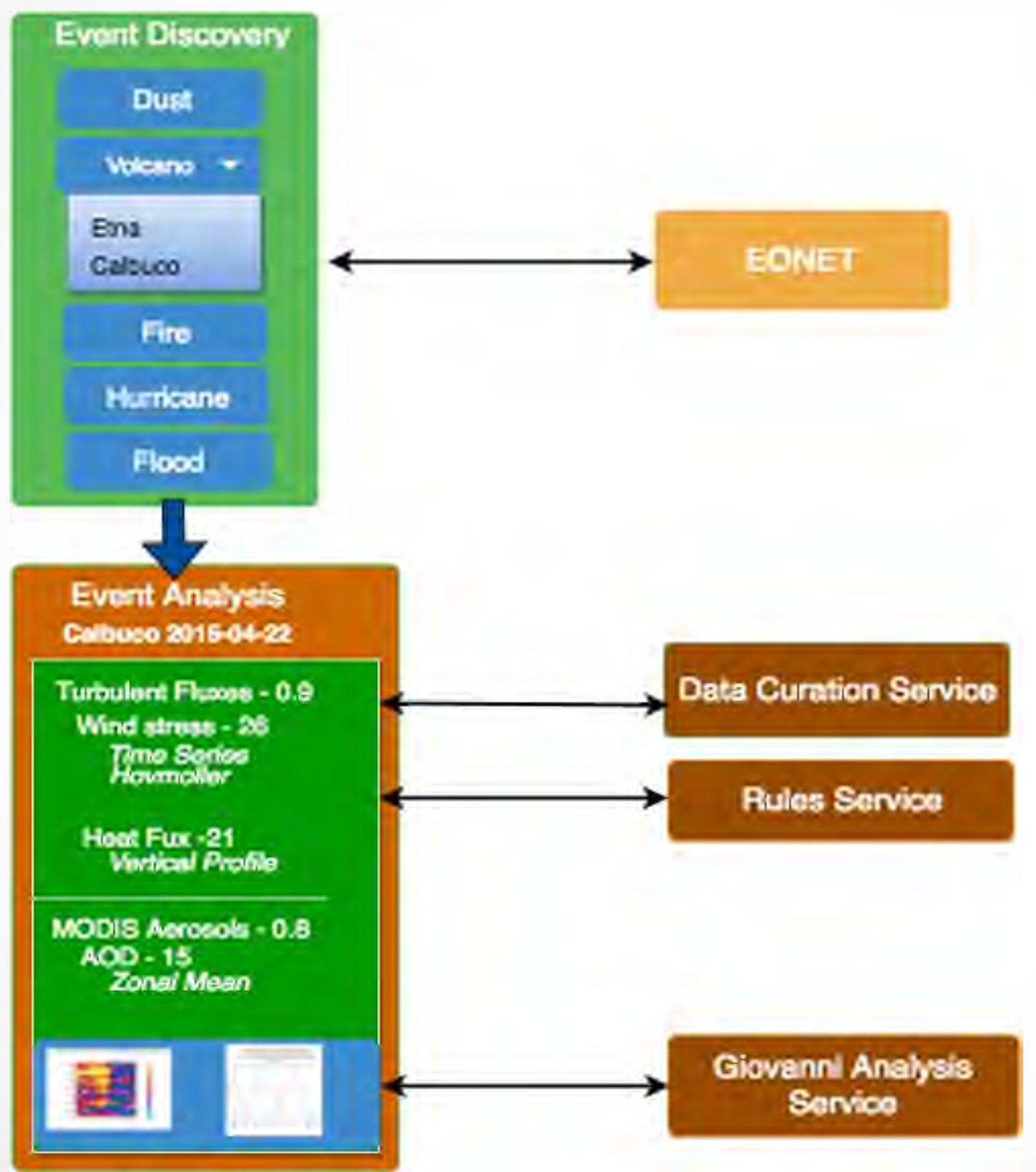

Event Analysis Workflow 


\section{DEMO}


Part 5: Image Retrieval 


\section{Image Retrieval}

- Goal: given an image of Earth science phenomenon retrieve similar images

- Challenge: "semantic gap"

- low-level image pixels and high-level semantic concepts perceived by humans 
"Deep" Architecture

- Features are key to recognition

- What about learning the features?

- Deep Learning

- Hierarchical Learning

- Mimics the human brain that is organized in a deep architecture

o Processes information through multiple stages of transformation and representation

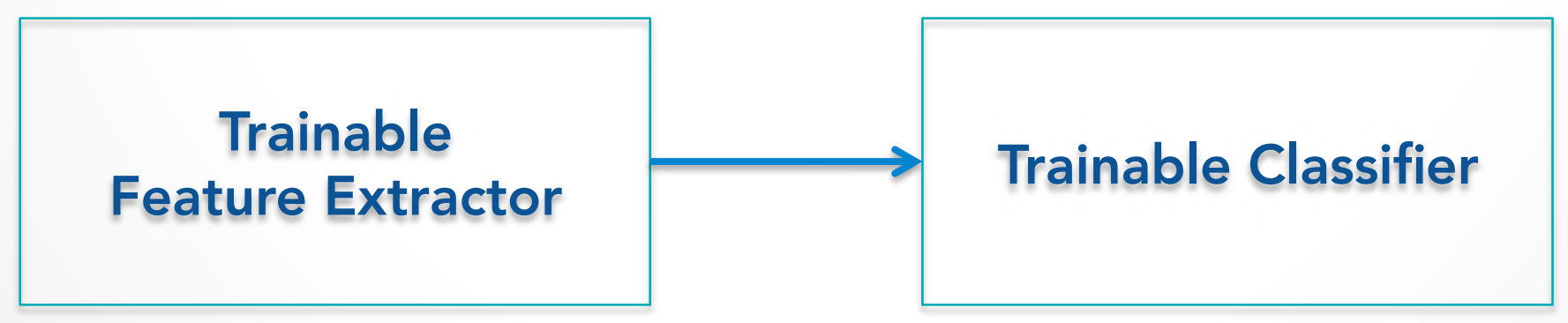

Convolutional Neural Network (CNN) - Applicable to Images 


\section{Transfer Learning}

- CNN requires large number of parameters

- Learning parameters from a few thousand training samples is unrealistic

- Transfer learning

- Use internal representation learned from one classification task to another

- AlexNet architecture - Krizhevsky et. al.

- Weights learned from ImageNet 1.3 million highresolution images

- State-of-the-art classification accuracy 


\section{Experiment: CNN Configuration}

Text

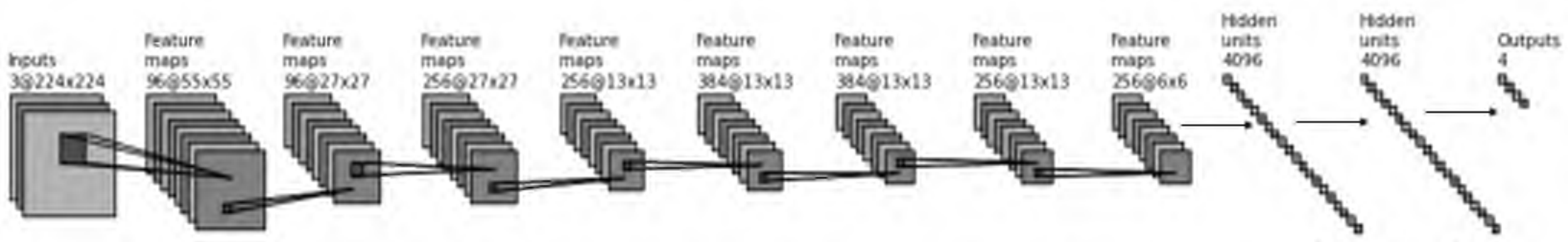

Convolution

Maxpooling Convolutisc

Masipoling Cerrvolution Cerrolution Convolution

Max-pooling

$3 \times 21 \times 11$ bernel $3 \times 3$ bornel $48 \times 5 \times 5$ bernel

$3 \times 3$ kemel $256 \times 3 \times 3$ kemel $192 \times 3 \times 3$ kemel $192 \times 3 \times 3$ knenel

3x 3 bernel

connected

Rully

connected

- AlexNet architecture

- Initialized weights with ImageNet trained model

- Adaptive learning rate

- GPU implementation 


\section{Experiment CNN - Visualization}

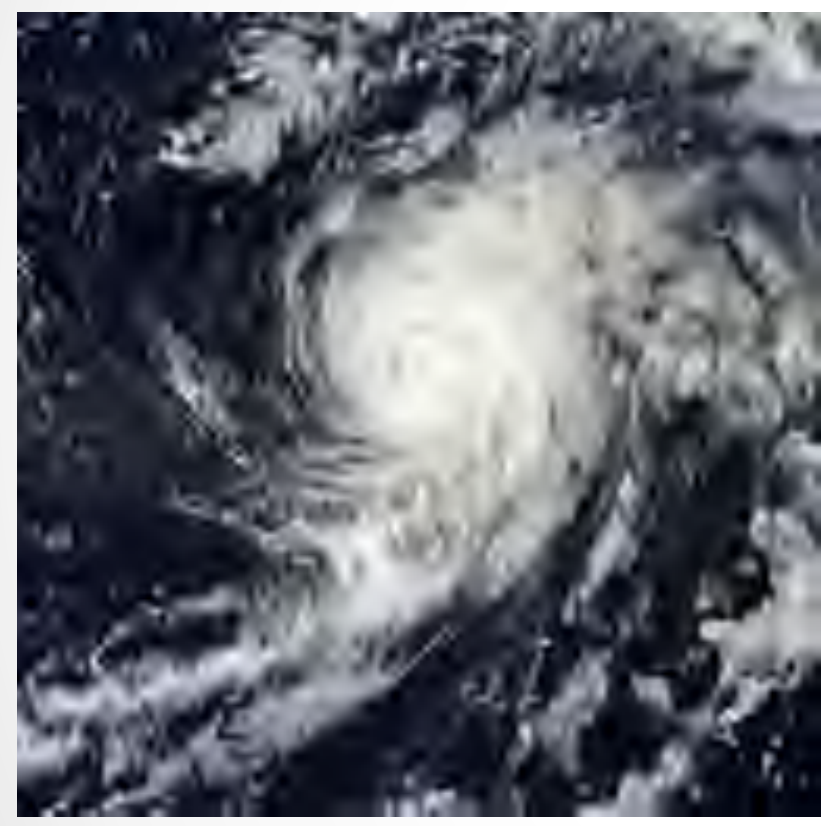

Input Image

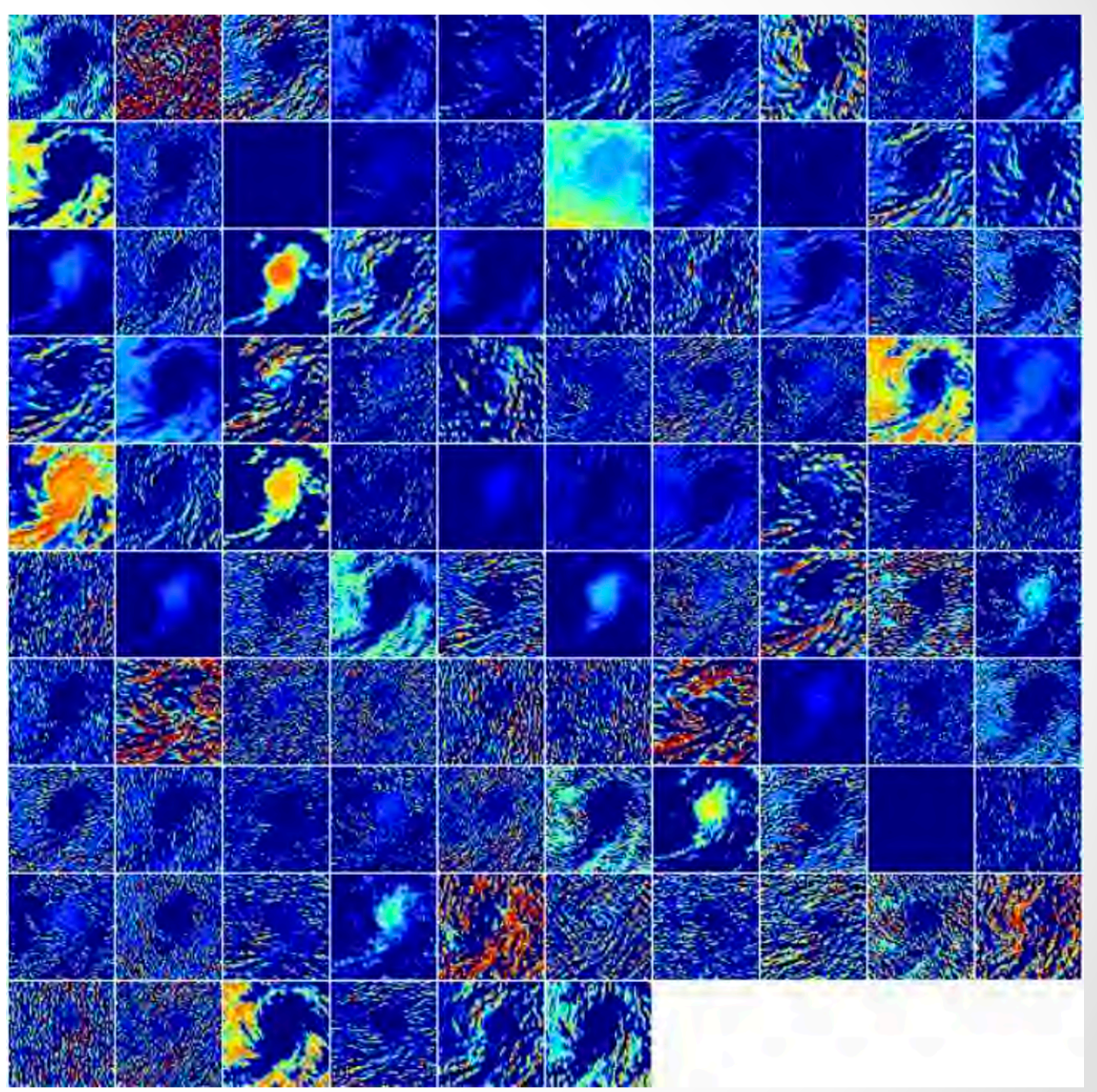

Feature Maps - Convolution Layer 1 


\section{Experiment CNN - Visualization}

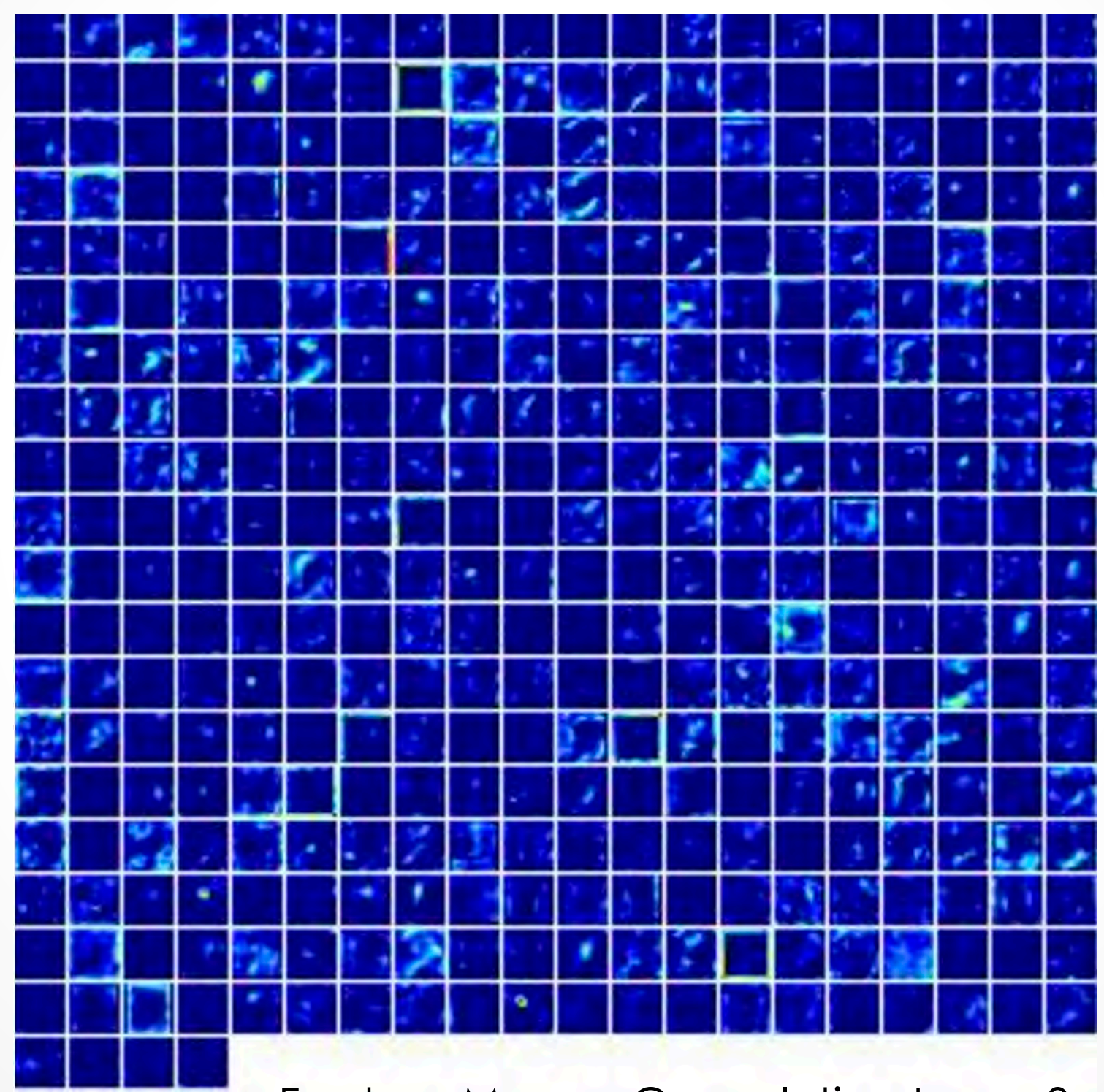

Feature Maps - Convolution Layer 3 


\section{Results: Confusion Matrix}

MODIS Rapid Response Test Images (Images are New to Trained CNN)

\begin{tabular}{|c|c|c|c|c|}
\hline True/Pred & Dust & Hurricane & Smoke & Other \\
\hline Dust & $\mathbf{2 8 7}$ & 8 & 32 & 33 \\
\hline Hurricane & 0 & $\mathbf{3 7 9}$ & 1 & 10 \\
\hline Smoke & 12 & 12 & $\mathbf{4 4 3}$ & 9 \\
\hline Other & 33 & 9 & 23 & $\mathbf{2 1 1}$ \\
\hline
\end{tabular}

Overall Accuracy $\mathbf{= 8 7 . 8 8 \%}$

Producer's Accuracy

Dust $86.45 \%$

Hurricane $92.89 \%$

Smoke $88.78 \%$

Other 80.23
User's Accuracy

Dust $79.72 \%$

Hurricane $97.18 \%$

Smoke $93.07 \%$

Other $76.45 \%$ 


\section{Results (MODIS Rapid Response)}

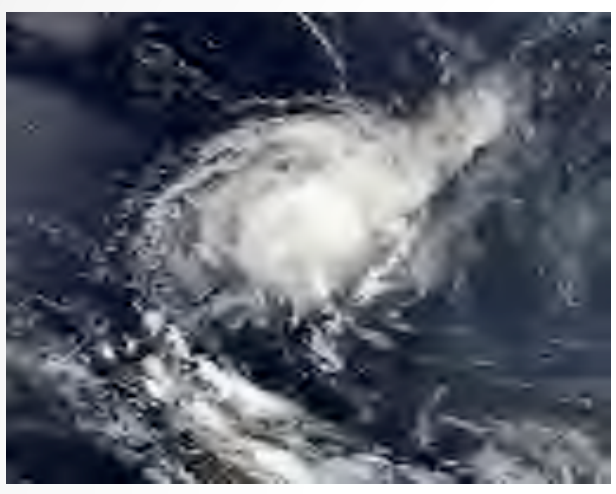

Hurricane - True Positive

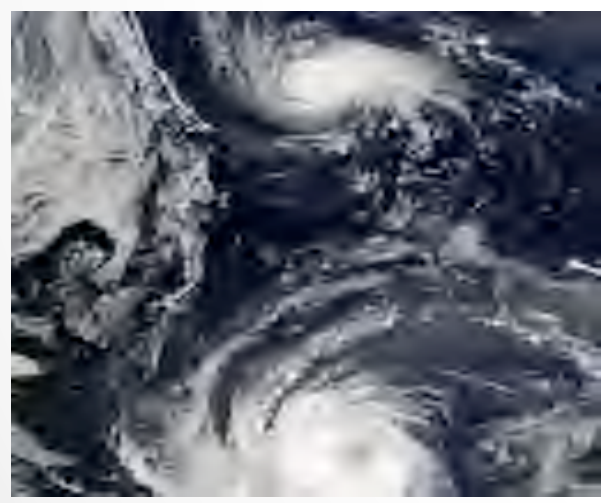

Hurricane - False Negative

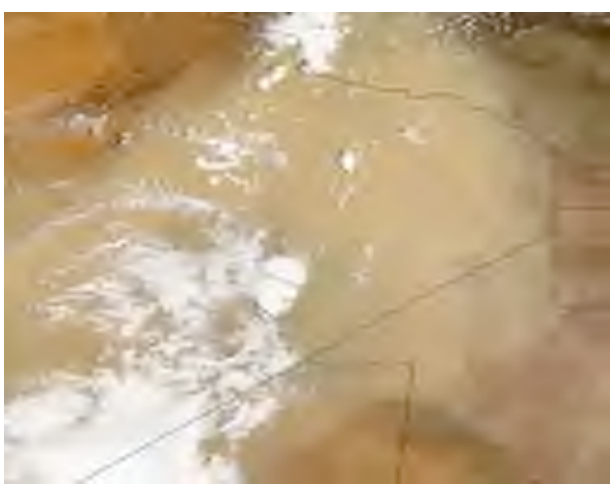

Dust - True Positive

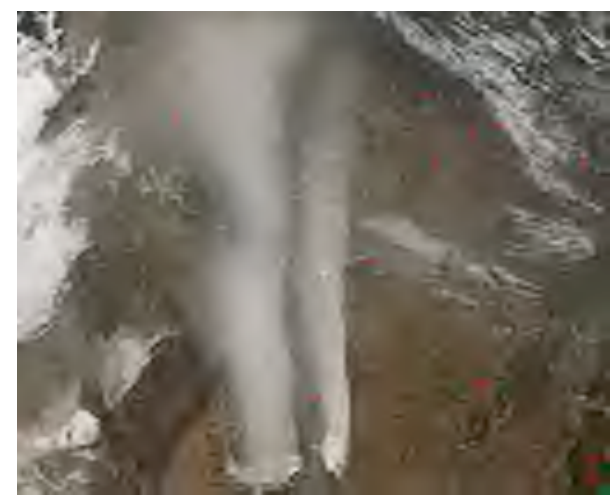

Dust - False Positive

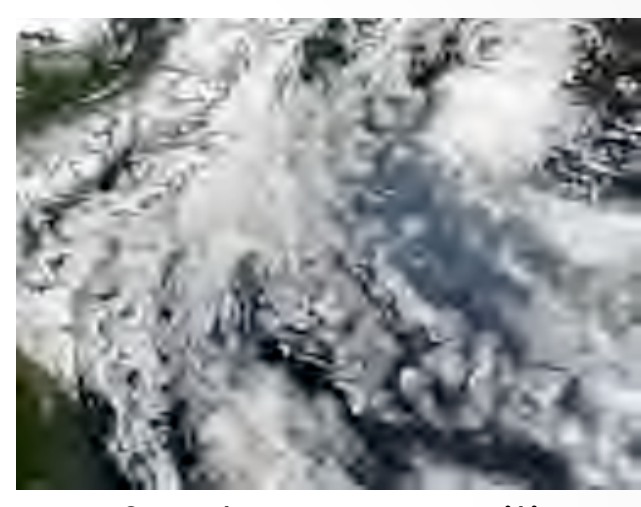

Smoke- True Positive

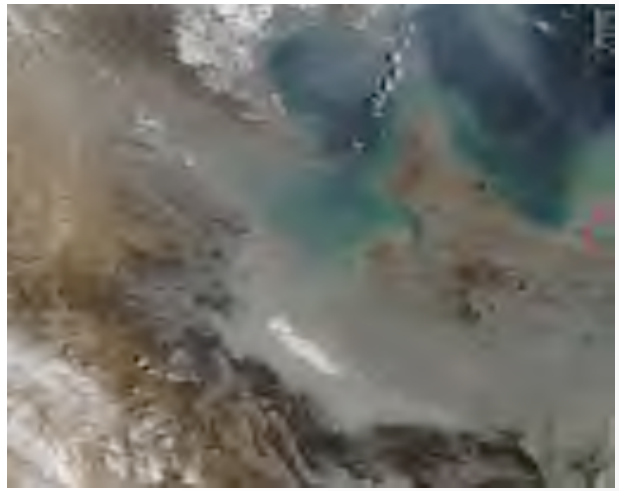

Smoke- False Positive 


\section{Applications: Enabling new science}

- Dust climatology - Collaboration with Sundar Christopher, UAH Atmospheric Science Professor

\begin{tabular}{|l|l|l|l|}
\hline True $\backslash$ Predicted & Dust & Other & Total \\
\hline Dust & $\mathbf{1 3 7 9}$ & 379 & 1758 \\
\hline Other & 260 & $\mathbf{4 9 3 2}$ & 5192 \\
\hline \multicolumn{4}{|c|}{ Confusion Matrix } \\
& 1639 & 5311 & 6950 \\
\hline
\end{tabular}

Validation
Accuracy $=\mathbf{9 1 \%}$

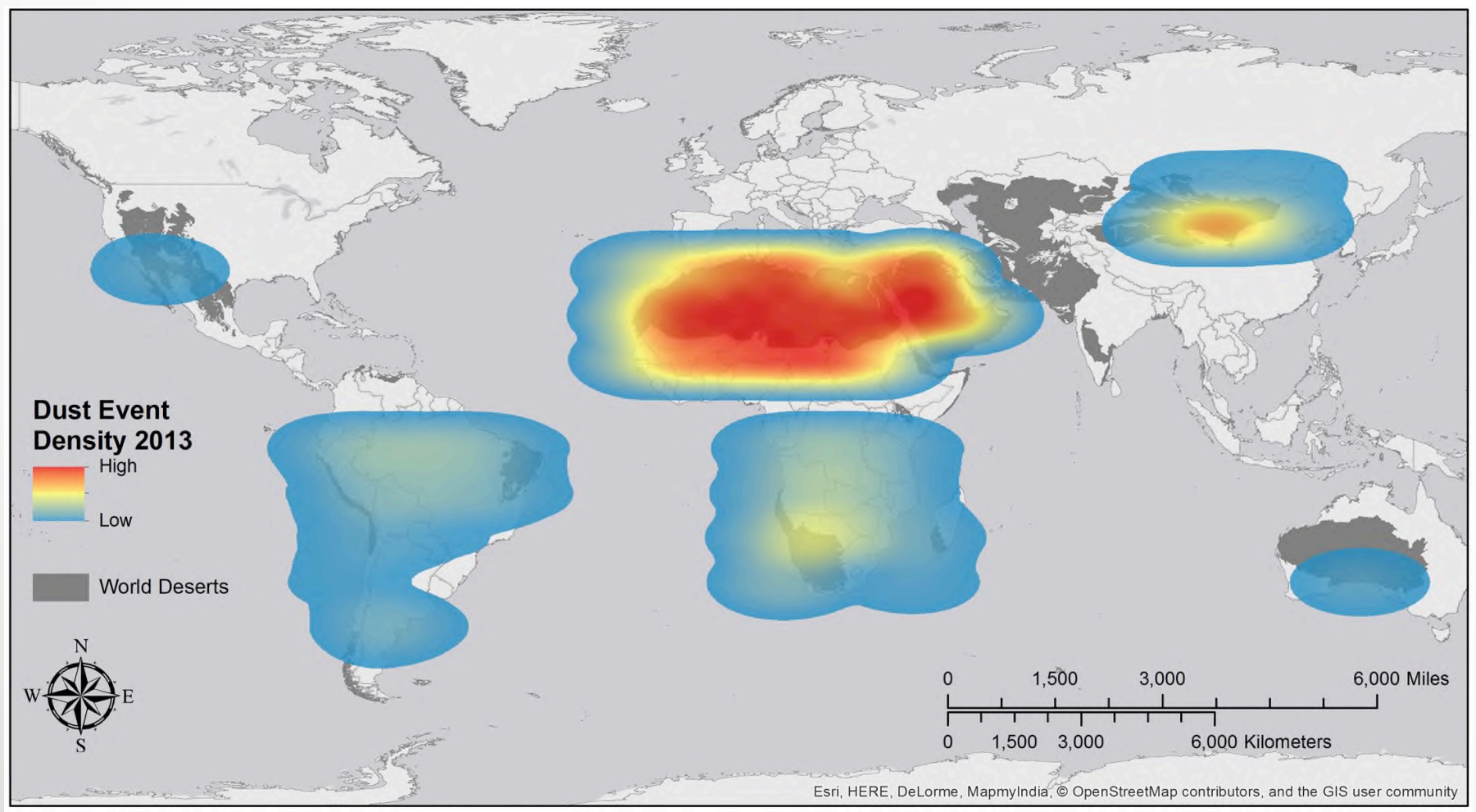

Based on GIBS 


\section{Applications: Improving forecast operations}

- Hurricane intensity estimation - Collaboration with Dan Cecil, NASA/MSFC Atmospheric Scie!

\begin{tabular}{|l|r|r|r|r|r|r|r|r|r|r|}
\hline TruelPredicted & td & ts & h1 & h2 & h3 & h4 & h5 & no_cat & total \\
\hline td & 3168 & 335 & 0 & 1 & 0 & 0 & 0 & 6 & $\mathbf{3 5 1 0}$ \\
\hline ts & 489 & 4823 & 159 & 5 & 11 & 3 & 6 & 0 & $\mathbf{5 4 9 6}$ \\
\hline h1 & 9 & 484 & 1158 & 92 & 20 & 6 & 1 & 0 & 1770 \\
\hline h2 & 3 & 76 & 214 & 513 & 145 & 4 & 0 & 5 & 960 \\
\hline h3 & 6 & 40 & 33 & 155 & 689 & 55 & 0 & 0 & 978 \\
\hline h4 & 1 & 18 & 17 & 12 & 142 & 810 & 32 & 0 & 1032 \\
\hline h5 & 2 & 2 & 0 & 0 & 27 & 59 & 216 & 0 & 306 \\
\hline no_cat & 22 & 0 & 0 & 0 & 0 & 0 & 0 & 32 & 54 \\
\hline
\end{tabular}

Overall Accuracy : 81 \%(Top 2 Probabilities 95.73\%)

Data: NRL Images, HURDAT

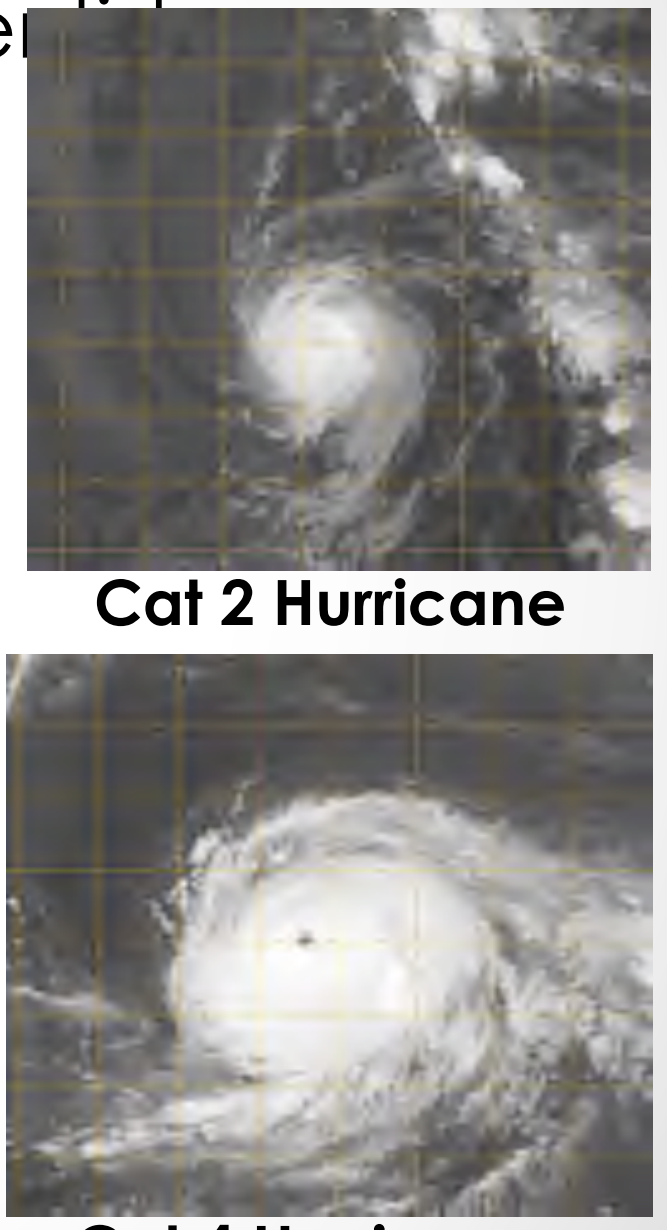

Cat 4 Hurricane 


\section{Ongoing Work}

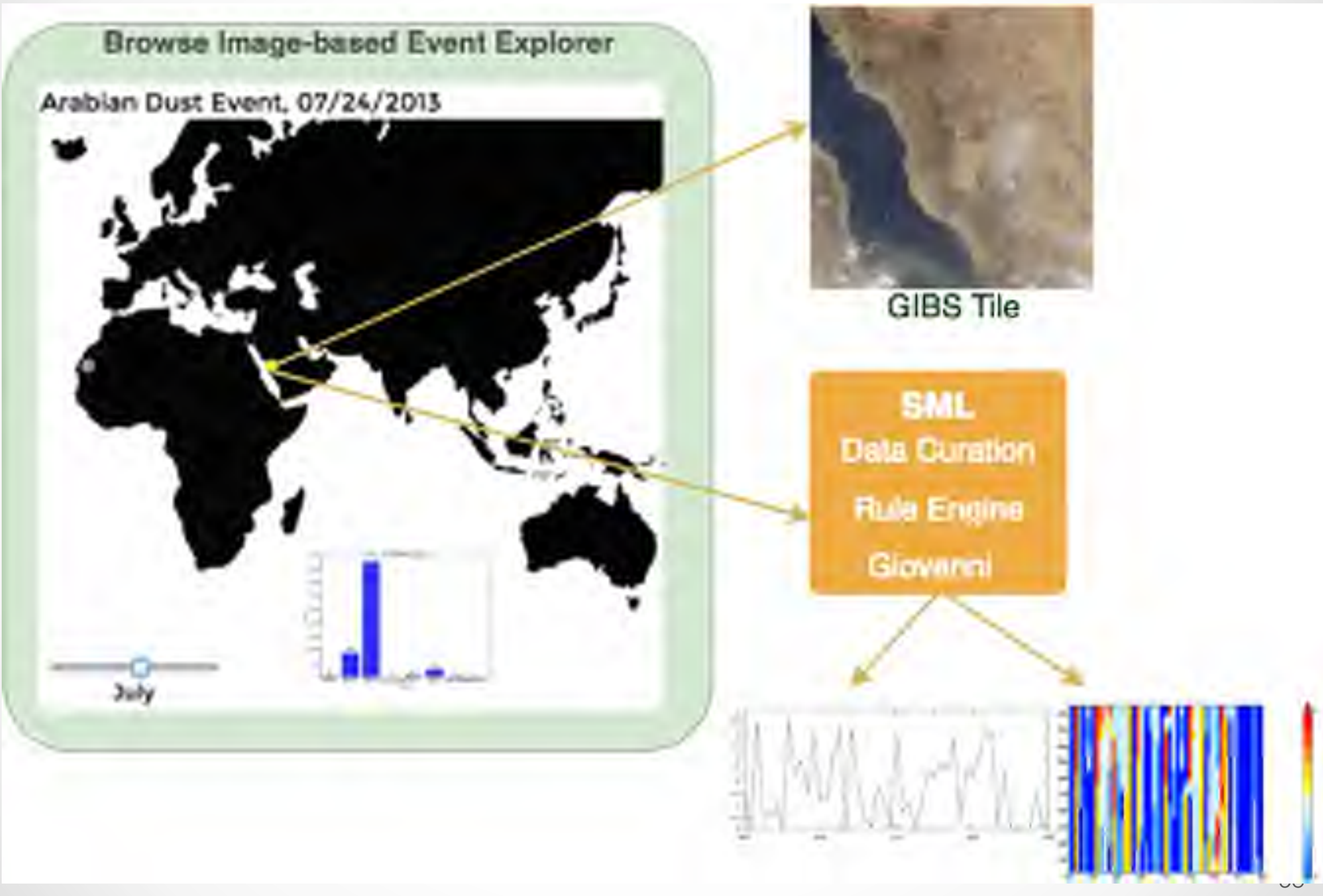




\section{Summary}

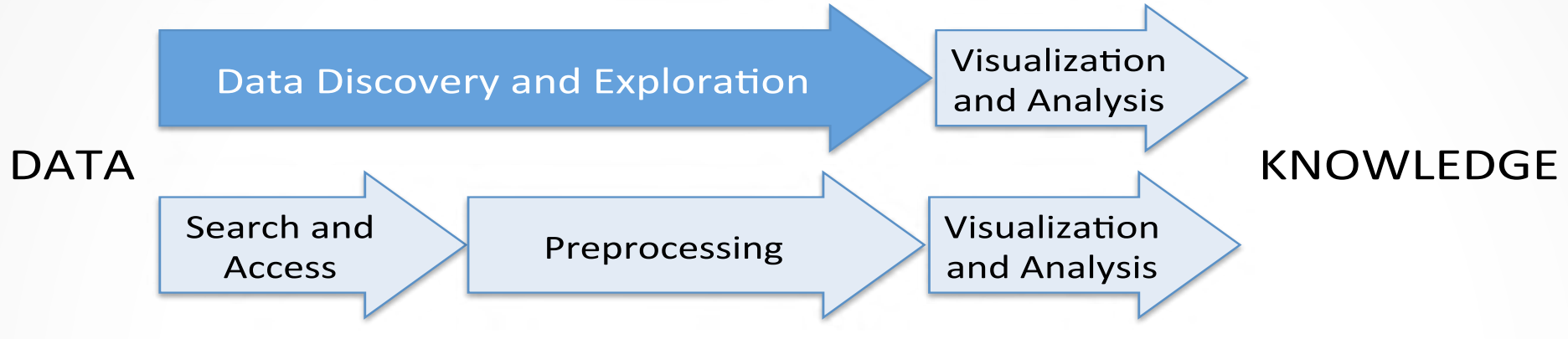

- Science data and information systems need to evolve to enable better data search, access and usability!

- Need operational services like - Data Curation Service, Rules Engine and Image Retrieval 


\section{Questions}

\section{Dr. Rahul Ramachandran}

Deputy Editor, Earth Science Informatics Journal Manager, Global Hydrology Resource Center NASA's Distributed Active Archive Center Earth Science Office (ZP 1 1) NASA / Marshall Space Flight Center Huntsville, Alabama 35812, USA

(w) 256.961 .7620

(c) 256.226 .6854

Orcld: orcid.org/0000-0002-0647-1941 www.linkedin.com/in/rramachandran05/ 